\title{
Adulthood Deficiency of the Insulin-like Growth Factor-1 Receptor in Hippocampal Neurons Impairs Cell Structure and Spatial Learning and Memory in Male and Not Female Mice
}

Abbreviated title: Reduced neuronal IGF-1 signaling impairs learning in mice

Cellas A. Hayes ${ }^{1}$, Erik L. Hodges ${ }^{1}$, Jessica P. Marshall ${ }^{1}$, Sreemathi Logan ${ }^{2,3}$, Julie A. Farley ${ }^{3}$, Daniel B. Owens ${ }^{3}$, William E. Sonntag ${ }^{3}$, Nicole M. Ashpole ${ }^{1,4, *}$

1. Department of BioMolecular Sciences, University of Mississippi School of Pharmacy, University of Mississippi, University, MS 38667

2. Department of Rehabilitation Sciences, University of Oklahoma Health Sciences Center, OK

3. Center for Geroscience and Healthy Brain Aging, Department of Biochemistry and Molecular Biology, University of Oklahoma Health Sciences Center, OK

4. Research Institute of Pharmaceutical Sciences, University of Mississippi School of Pharmacy, University of Mississippi, University, MS 38677

*Corresponding author: nmashpol@olemiss.edu

Declarations of interest: none. 


\begin{abstract}
Reductions in insulin-like growth factor-1 (IGF-1) are associated with cognitive impairment and increased risk of neurodegenerative disease in advanced age. In mouse models, reduced IGF-1 early-in-life leads to memory impairments and synaptic dysfunction; however, these models are limited by systemic reductions in IGF-1. We hypothesized that IGF-1 continues to promote hippocampal neuron structure and function after development, and as such, the loss of IGF-1 signaling in adult neurons would lead to impaired spatial learning and memory. To test this, the IGF-1 receptor (IGF-1R) was genetically targeted in hippocampal neurons of adult male and female mice. Male mice deficient in neuronal IGF-1R exhibited spatial learning impairments as evidenced by increased pathlength and errors in the radial arm water maze. No differences in learning and memory were observed in female mice. Golgi-Cox staining revealed a reduced number of dendritic boutons of neurons the CA1 region of the hippocampus in male mice. Decreased MAPK and increased ROCK activity were also observed in these tissues. In vitro studies revealed that impaired neurite outgrowth due to inhibited IGF-1R signaling could be rescued by pharmacological inhibitors of ROCK. However, ROCK inhibition in neuronal IGF-1Rdeficient mice did not fully rescue learning impairments or bouton numbers. Together, our study highlights that IGF-1 continues to support spatial learning and memory and neuronal structure in adulthood.
\end{abstract}

Keywords: IGF-1, somatomedin C, cognition, sex-specific, neuronal, hippocampus

\title{
INTRODUCTION
}

Insulin-like growth factor-1 (IGF-1) promotes of brain development and pubertal growth. While much focus is placed on the impact of IGF-1 during the developmental phases of life, the growth hormone (GH) and IGF-1 signaling axis are implicated in critical behavioral and cellular functions throughout the lifespan. In fact, one of the hallmarks of aging is the reduction in the GH/IGF-1 signaling (Ashpole et al., 2015b; Lopez-Otin et al., 2013). Circulating IGF-1 levels are reduced 30-60\% in elderly human and animal models (Florini et al., 1985; Johanson and Blizzard, 1981; Rudman et al., 1981). This decrease is temporally associated with the onset of cognitive decline and increased risk of neurodegenerative diseases and dementias (Arwert et al., 2006; Deijen et al., 2011; Frater et al., 2018; Sonntag et al., 2005b; Trejo et al., 2007). Importantly, supplementation of IGF-1 has been shown to reverse cognitive impairment in aged animals (Markowska et al., 1998), suggesting that maintenance of the IGF-1 signaling cascade remains important for neuronal function after development. Recombinant IGF-1 is FDA approved for the treatment of early-life growth deficiencies, however its use is limited to this developmental growth window and has not been tested in cognitively-impaired, elderly humans. As an anabolic hormone associated with increased cancer risk, systemic administration of exogenous IGF-1 is not an ideal therapeutic for adult and elderly individuals (Shanmugalingam et al., 2016; Sonntag et al., 2005a). Instead, it may be advantageous to selectively target one of the downstream signal transduction cascades that are dysregulated when IGF-1 levels decline in advanced age. In order to do this, a better mechanistic understanding of the influence of IGF-1 on cognitive behaviors in adulthood is needed. 
Interruptions in the IGF-1 signaling cascades lead to significant impairments in long-term potentiation and learning and memory (Cao et al., 2011; D'Mello et al., 1997; Gazit et al., 2016; Glasper et al., 2010; Maher et al., 2006). In the developing brain, the beneficial effects of IGF-1 are thought to be mediated by pro-survival and pro-growth pathways. Activation of the IGF-1 receptor (IGF-1R) and the downstream PI3K, Akt, and MAPK signaling cascades enhance neuronal survival, growth, and excitability (Cheng et al., 2003; Scolnick et al., 2008; Trejo et al., 2007). Little is known about the effects of IGF-1 on growth-limiting pathways. In fact, several intracellular signaling cascades limit neuronal structure throughout the lifespan, including the RhoA/Rho-associated coiled-coil containing protein kinase (ROCK) pathway, which stabilizes neurofilament rearrangement (Bito et al., 2000; Hirose et al., 1998). Increased RhoA/ROCK is associated with spine retraction, synapse elimination, decreased neuronal excitability, and impaired learning and memory (Dash et al., 2004; Lamprecht et al., 2002; Sunico et al., 2010). Expression and activity of the RhoA/ROCK pathway are upregulated in advanced age and are further upregulated in cognitively-impaired aged animals (VanGuilder Starkey et al., 2013). In aged rats and mouse models of Alzheimer's Disease and intellectual disability, ROCK inhibition rescues spatial learning and memory impairments (Busti et al., 2020; Guo et al., 2020; Huentelman et al., 2009; Meziane et al., 2016; Yu et al., 2017). If IGF-1 regulates ROCK activity, then the agerelated loss of IGF-1 could account for the upregulation of this growth-limiting pathway.

Given the temporal associations with reduced circulating IGF-1, increased ROCK, and impaired cognition, we hypothesized that the loss of IGF-1 in adulthood leads to decreased growthpromoting signals as well as increased growth-restricting signals, which ultimately causes loss of neuronal structure inherently leading to learning impairments. The common mammalian model organisms in which IGF-1 signaling is altered involve reducing hepatic production of IGF-1, which is responsible for releasing IGF-1 into circulation. This alters systemic IGF-1 signaling, thereby confounding our understanding of the cell-specific activities. Indeed, reductions in circulating IGF-1 alters the structure and function of several cell types, including astrocytes, microglia, and the cerebrovasculature (Grinberg et al., 2013; Logan et al., 2018; Park et al., 2011; Prabhu et al., 2019; Tarantini et al., 2016a). Most IGF-1-deficient animal models also have reduced IGF-1 levels throughout both development and adulthood, which may instigate compensatory effects that confound interpretation. To date, studies have not delineated whether IGF-1 continues to regulate learning and memory by acting specifically on neurons after development. To address this, we utilized genetic tools to specifically target IGF-1R signaling in hippocampal neurons in adulthood and then assessed the molecular, cellular, and behavioral changes induced in the months following knock-out.

\section{MATERALS AND METHODS}

Animals: All procedures were approved by the Institutional Animal Use and Care Committees of the University of Mississippi and the University of Oklahoma Health Sciences Center. IGFR ${ }^{\text {f/f }}$ were purchased from Jackson laboratories (B6;129-Igf1rtm2Arge/J) and bred in house to develop experimental cohorts ( $\mathrm{N}=128$ experimental mice). Spatial learning and memory was assessed at both research sites to verify reproducibility following lab relocation. These mice express Lox P sites surrounding exon 3, which encodes a key region of the ligand-binding domain of IGF-1R. 
All mice were genotyped to verify igfrfff, using the primers and PCR protocols designed by Jackson laboratories, as previously reported (Prabhu et al., 2019). At the University of Oklahoma Health Sciences Center, mice were housed under specific pathogen-free barrier conditions in the Rodent Barrier Facility under a controlled photoperiod (12 h light; 12hdark) with unlimited access to water and standard AIN-93G diet food (ad libitum). At the University of Mississippi, mice were housed in ventilated racks (Lab Products, Inc. RAIR Envirogaurd ventilated cages) with enriched bedding under a 12:12 hour photoperiod, with 5053 Pico Lab chow and water ad libitum. Block randomization was utilized to ensure balanced group size and treatment groups were separated by cages. Male and female cohorts were treated independently. Following behavioral analysis, mice were euthanized via $\mathrm{CO}^{2}$ asphyxiation for ex vivo tissue isolation and analysis.

Maintenance of igfrff homozygosity was verified in all offspring after weaning (3-5 mice per cage). Following 2-3mm tail removal, DNA was extracted using the RED Extract-N-Amp Tissue PCR XNAT, following manufacturer's instructions (Sigma). The recommended IGFR flox primers and PCR protocols designed by Jackson laboratories were utilized (primers purchased from Sigma). PCR products were run on a 1.5\% agarose gel stained with green glow (Denville) at $120 \mathrm{~V}$ in $1 \mathrm{XTB}$ for 1 hour. UV imaging identified PCR products. All gels contained a 100bp DNA ladder (ThermoFisher), an IGFR ${ }^{f / f}$ control lane, and an IGFR ${ }^{+/+}$control lane.

Primary neuron cultures were developed using tissue from post-natal (P1) igfrff mice. Male and female pups were not separated during isolation. Neuron cultures were also developed using tissue from embryonic (E-19) and post-natal (P1) Sprague Dawley rats. Timed pregnant rat dams were purchased from Envigo and were euthanized along with the pups.

Hippocampal Adeno-Associated Virus Injections: At three months of age, cohorts of male and female igf $^{f l / f l}$ mice received stereotaxic injections of AAV9-Syn-Cre or control AAV9-Syn-GFP (purchased purified virus from Penn Viral Vector Core) in the dentate gyrus and CA1 subregions of the hippocampus (Barbash et al., 2013; Stoica et al., 2013). Mice were anesthetized with ketamine/xylazine or with 5\% isoflurane and the anesthetic plane was verified with a toe pinch. Mice were placed on a heating pad, EMLA cream (Lidocaine (2.5\%) and Prilocaine Cream (2.5\%), USP) was applied to the stereotactic ear bars, and a dab of Puralube was placed on each eye. Saline $(1 \mathrm{~mL})$ was subcutaneously injected to prevent dehydration. The topical area of the cranium was scrubbed with $1 \%$ chlorohexidine or $70 \%$ ethanol and the hair was trimmed. A $5-8 \mathrm{~mm}$ incision exposed the sagittal suture, the skull was leveled, and bregma and lambda measurements were used to determine injection coordinates (posterior $-0.28 \mathrm{~mm}$, medial/lateral $+/-0.25$ ). A high speed microdrill was used to puncture the skull at the calculated position and a Hamilton syringe was guided to the injection depth ( -0.27 for CA1 and $-0.3 \mathrm{~mm}$ for $\mathrm{DG})$. Bilateral injections were performed in all mice with each hippocampal hemisphere receiving a total of $1 \mu$ diluted virus $(0.5 \mu \mathrm{l}$ per injection depth, administered at $0.1 \mu \mathrm{ls} / 10$ seconds). Bone wax (Surgical Specialties) was applied to the injection holes and the incision was then mattress sutured (Nylon sutures). Meloxicam or Bupivicaine (Henry Shrien) was administered for pain (immediately and as needed with 48 hours of procedure).

Hydroxyfasudil Osmotic Pump Implantation: A cohort of mice received hydroxyfasudil (Sigma) or control DMSO/ddH2O for 14 days via implanted subcutaneous mini osmotic pumps 
(Alzet). Pumps were equilibrated in 0.9\% saline overnight and loaded using the manufacturer's instructions. Mice were weighed to determine $10 \mathrm{mg} / \mathrm{kg}$ dosing of hydroxyfasudil (diluted in 18\% DMSO and $82 \%$ ddH2O) in $200 \mu \mathrm{l}$ buffer across 14 days. Groups were block randomized to receive hydroxyfasudil or vehicle control; however, 6 controls (*IGFR-WT) did not receive pumps (all remaining mice within that experimental cohort did). Mice were anesthetized using $5 \%$ isoflurane and the osmotic pump was inserted under the skin at the nape of the neck. The incision site was mattress sutured and lidocaine was administered topically on the wound. Pumps remained in place throughout the behavioral assessment. No adverse effects were noted.

Quantitative PCR: Total RNA was isolated using the RNAeasy mini kit (Qiagen) and converted to cDNA using High-Capacity RNA-to-cDNA kit (Applied Biosystems). RT-PCR was performed using TaqMan Universal PCR Master Mis (Life Technologies) and TaqMan validated primers on the CFX Connect Real-time PCR detection system (Bio-Rad). Results were normalized to housekeeping genes, HPRT and B2M. The experimenter was blinded to treatment group during experimentation, and the assignment code was revealed for delta-delta CT analysis.

Radial Arm Water Maze: Two months following viral injection, spatial learning and memory was assessed in a flooded -eight-arm maze with a submerged escape platform and spatial clues. Water (5-7cm depth) was mixed with white food coloring to create an opaque color to hide the safety platform. Mice were acclimated to the escape platform before beginning the acquisition phase of the maze, which consisted of 24 training trials (8 trials per day for 3 consecutive days). On day 10 , memory was assessed in a 60 -second probe trial. The platform was then moved to an alternate arm for a reversal phase where extinction and re-learning were observed for an additional 8 trials. Total pathlength to escape platform, latency to escape, the number of errors made, and velocity were calculated using Ethovision Software (Noldus Information Technology Inc., Leesbur, VA, USA). Six male mice that did not undergo stereotaxic AAV injection and were included as an additional treatment control group in this maze.

Novel Object Relocation and Recognition: Assessment of working memory with the novel object (NO) and novel location (NL) assay was carried out over 5 exploration trials in a whitewalled open arena containing 2 objects. Each trial was 5 minutes in length, with a 5-minute intertrial interval. Trials 1, 2, and 3 were used to familiarize the mouse with the arena. In trial 4, the object was relocated (NL) and in trial 5, the object was replaced (NO). The time spent at each object was used to calculate the discrimination index for NL (time spent at NL/(time spent at NL + time spent at F3)) and $\mathrm{NO}$ (time spent at $\mathrm{NO} /($ time spent at $\mathrm{NO}+$ time spent at F3)) using Ethovision Software (Noldus Information Technology Inc.).

Western Blot: Hippocampal tissue and primary neuron cultures were lysed in RIPA buffer (Fisher) with protease and phosphatase inhibitors (Calbiochem, Roche). Protein concentration in the lysates was calculated using an RC/DC Assay, per manufacturer's recommendations (BioRad). Protein (10-20 $\mu \mathrm{g})$ was denatured and loaded into 4-12\% precast bis-tris gels (Invitrogen, Thermo Fisher). Following electrophoresis, gels were transferred to nitrocellulose membranes, blocked in $5 \%$ BSA in 1XTBST, incubated with primary antibody, washed, and incubated with secondary antibodies. All antibodies, dilutions, and RRIDs are described in Supplemental Table 1. Blots were imaged and analyzed on the Licor Odyssey CLx. 
Golgi Staining: A sagittal hemisphere of the brain was stained using the FD Rapid Golgi Stain Kit (FD Neuro Technologies, Inc.), following the manufacturer's recommendations. Due to equipment procurement between studies, female specimens were sliced at $100 \mu \mathrm{m}$ on a cryostat (Leica), while male specimens were sliced at $250 \mu \mathrm{m}$ after being solidified in $3 \%$ agarose in ddH20 on a vibratome (Precision Instruments) with an oscillation speed of 6.9 and speed of 1.9. Brightfield imaging was performed on a Nikon Ti2-E inverted microscope with a 60x objective. Z-stacked images were acquired and a $20 \mu \mathrm{m}$ line was drawn on dendrites selected at random to quantify the number of boutons across a controlled length. Two dendrites per field were selected by a blinded observer. Images were taken of both sub-regions, DG and CA1, for separate quantification.

Primary neuron cultures: Hippocampal and/or mixed hippocampal/cortical tissue was isolated from igfrfff mice or wild-type Sprague Dawley rats to establish primary cultures of neurons, following our previously established protocols (Ashpole and Hudmon, 2011). Tissue was enzymatically digested with Papain (Worthington Biochemical Corporation) at $37^{\circ} \mathrm{C}$ for $20-30$ minutes, mechanically triturated, and filtered. Cells were pelleted and resuspended in fresh neuronal plating media Neurobasal media (Life Technologies), 5\% FBS (Cellgro or Sigma), 1X Glutamax (Life Technologies), and 1X N2 Supplement (Life Technologies). The next day, media is changed to neuronal growth media (Neurobasal media, 1X B27 (Life Technologies), 1X Penicillin/ Streptomycin, and $1 \mathrm{X}$ Glutamax), and is maintained in this media with mitotic inhibitors until experimental use. For experiments were neurons where IGF-1R is genetically reduced, AAV9-Syn-Cre and control AAV9-Syn-GFP was transduced at day 5 of culturing. For all pharmacological treatments, drugs were diluted in fresh media and administered on days 8-10 of culture, and experimental endpoints were 24 hours later.

Sholl analysis: Hippocampal neurons were grown on coverslips and transfected with CMVtdTomato (Addgene) between days 2 and 5 of culture. Treatments were administered between days 8-10 and 24 hours later, neurons were fixed in 4\% paraformaldehyde in phosphate buffer, pH 8.0. Coverslips were washed with 1XPBS and mounted using Prolong Gold Antifade with DAPI. Transfected neurons were selected at random by a blinded observer and imaged using a Nikon Ti2E inverted microscope with a 20x objective and 540/70 excitation/emission filter. Multiple fields (6-10) were selected at random on each coverslip (8-10) for analysis with Neurolucida (MBF Biosciences) or ImageJ (Fuji) Sholl plug-ins. The average area under the curve for each coverslip was interpolated using SigmaPlot v13 (Systat).

ROCK Activity Assay: Hippocampal tissue or $10 \mathrm{~cm}$ dishes of primary mixed cortical/hippocampal neurons were lysed in RIPA buffer (Fisher) containing 1X protease and phosphatase inhibitors (Calbiochem, Perkin Elmer) for use in the Rho-associated Kinase Activity Assay (Millipore). The manufacturer's recommendations were followed for all steps of the assay. This assay does not differentiate ROCK-1 and ROCK-2 activity.

RhoA Activation Assay: GTP-bound RhoA was quantified using the RhoA Pull-down Activation Assay Kit (Cytoskeleton, Inc), following the manufacturer's recommendations. Mixed neuronal cultures were treated with pharmacological inhibitors for 24 hours and subsequently lysed in the 
proprietary lysis buffer. GST-tagged Rhotekin-RBD beads were incubated in the lysate prior to washing, eluting, and western blotting with the materials provided in the kit.

Experimental Design and Statistical Analysis: All analysis and graph generation was performed using Sigma Plot v13 software and/or R Version 64 3.6.1 in addition, to RStudio with package lmerTest version 3.1-0. For comparisons of two treatment groups (WT vs KO or Vehicle v PPP), a Students unpaired t-test was utilized and normality was assumed. To compare concentrationdependence, a one-way ANOVA with posthoc Dunnett's test vs vehicle control was performed. Many experiments were set as 2x2 factor designs (genetic treatment and pharmacological treatment), thus two-way ANOVA with posthoc Fishers LSD or Bonferonni were utilized. Behavioral assays with multiple trials were set as two-way repeated-measures ANOVAs. Male and female cohorts were independently assessed and direct comparisons were not performed. Behavioral experiments were constructed to detect a $20 \%$ difference between treatment groups, with a power of 0.8 using a type 1 error rate of 0.05 . Based on preliminary data, we estimated $n=8$ mice were needed per treatment group. All data were expressed with mean $+/$ - standard error and $\mathrm{p}<0.05$ was used to define statistical significance. The statistical comparisons that accompany our experimental design and working hypotheses used are defined in Figure Legends. All p values and final sample sizes can be found in Supplemental Table 2.

All mice and neuron images were coded to blind experimenter to treatment groups during testing. Neurons were selected at random for structural studies, with multiple cells per brain slice or coverslip assessed for the rigorous representation of the data. No mice were excluded from the behavioral and/or molecular studies; however, 3 mice did die during hippocampal injection surgery.

\section{RESULTS}

\section{Learning impairments with neuronal IGFR deficiency}

Hippocampal neuron-specific IGF-1R deletion (*IGFR-KO) was induced in male and female igfrfff mice at 3-4 months of age via stereotaxic injections of Cre recombinase encoding viral vectors (AAV9-Syn-Cre or control AAV9-Syn-GFP) (Figure 1A). mRNA expression of IGFR, IGF-1, and other growth-promoting signals was quantified in the dorsal hippocampus to verify specific reductions in the floxed region of IGF-1R exon 3. IGF-1R was reduced by $39.6 \%$ in these neuron-specific *IGFR-KO male and female mice, compared to GFP-transduced controls (*IGFRWT) (Figure 1B, $\mathrm{p}=0.033, \mathrm{n}=9$ ). No significant differences in the expression of growth hormone receptor (GHR), insulin receptor (InsR), or IGF-1 were observed in the hippocampi of *IGFR-KO mice (Figure 1B, $\mathrm{p}>0.05, \mathrm{n}=9$ ). Details of exact $\mathrm{p}$-value comparisons throughout the entire result section are described in Supplemental Table 1.

Two to three months following knockout, spatial learning/memory was assessed in the radial arm water maze. *IGFR-WT and *IGFR-KO mice both learned to locate the escape platform over the three-day training phase, as indicated by a reduction in the total pathlength traveled to reach the escape platform (Figure 1C) and the total number of errors made in finding the platform (Figure 1E). However, *IGFR-KOs traveled significantly more and made more errors throughout the learning phase than controls (Figure 1C, $p=0.0002$; Figure 1E, $p=0.018$; $n=10,11$ ). When 
memory extinction and relearning was assessed in a reversal phase of the maze, male *IGFR-KO mice again traveled significantly more and made more errors than controls (Figure 1D, $\mathrm{p}=0.0017$; Figure 1F, $\mathrm{p}=0.011, \mathrm{n}=10,11)$. *IGFR-WT controls performed similarly to mice that had not undergone AAV injection, suggesting the surgical procedure alone did not alter spatial learning and memory (Supplemental Figure 1A)

Unlike males, female *IGFR-KO mice did not show significant impairments in spatial learning and memory compared to *IGFR-WT controls. Both groups of females exhibited similar reductions in pathlength and the number of errors over training days; no between-group differences were observed and no differences were noted in the reversal phase one week later (Supplemental Figure 1B, $\mathrm{p}>0.05, \mathrm{n}=9,10$ ).

In addition to testing spatial learning and memory, working memory was assessed in the novel object replacement and relocation task. No significant difference in exploration or novelty discrimination was observed in male *IGFR-KOs (Figure 1G-I, p>0.05, n=8,9) or female *IGFRKOs; however, *IGF-1R KO females explored the arena significantly more than female *IGFRWT females (Supplemental Figure 1C, $\mathrm{p}<0.01$, $\mathrm{n}=10$ ).

\section{Structure changes in Neurons Deficient in IGF-1R}

Following behavioral analysis, the neuronal structure within the CA1 region of the hippocampus was visualized using Golgi-Cox staining (Figure 2A-B) and synaptic bouton density was subsequently quantified. No difference in total neurite number or length was observed using Sholl analysis (area under the curve $145 \pm 37.2$ control vs $110 \pm 18.5 *$ IGFR-KO, p=0.51). Male *IGFRKO mice did have fewer CA1 synaptic boutons than controls (Figure 2B, p=0.0002, n=9,12). Similar to the behavioral outcomes, no difference in synaptic bouton number was observed in female *IGFR-KOs (Supplemental Figure 1D, $p=0.18, n=7,8$ ). While the bouton number in the CA1 was significantly reduced in male mice, no significant differences in total protein levels of synaptophysin or PSD-95 were seen in full dorsal hippocampal lysates (Supplemental Figure 1EF).

Changes in neuronal structure could also be noted in 8-10-day old primary hippocampal neuron cultures when IGFR signaling was reduced. Increasing concentrations of the small molecule IGFR inhibitor, picropodophyllin toxin (PPP), led to a concentration-dependent decrease in neurite complexity (Figure 2C-E). Note, neuron cultures were not stratified by sex as tissues were combined during isolation. Sholl analysis revealed a significant reduction in neurite length and complexity with $0.50 \mu \mathrm{M}-5 \mu \mathrm{M}$ PPP, compared to DMSO control (Figure 2E-F, $\mathrm{p}<0.05, \mathrm{n}=8$ ). Concentrations above $5 \mu \mathrm{M}$ were neurotoxic.

\section{Reduced MAPK and Increased ROCK with Neuronal IGFR Deficiency}

Because male mice exhibited behavioral and cellular alterations when neuronal IGF-1R was reduced, the activation of downstream kinase signaling cascades within the hippocampal tissue was then assessed. As anticipated, phosphorylated MAPK was significantly reduced in *IGFRKO hippocampi (Figure 3A, p=0.009, n=8,12). Conversely, ROCK activity was significantly 
increased (Figure 3B, $\mathrm{p}=0.025, \mathrm{n}=6,7$ ). Downstream of ROCK, the ratio of phosphocofilin to total cofilin of *IGFR-KOs was trending but did not reach significance (Figure 3C, p=0.08, n= 7,8).

Genetic reduction of IGF-1R in cultured neurons resulted in increased ROCK activity in the presence of exogenous IGF-1 in the media (Figure 3D, n=5-6). A two-way ANOVA comparison revealed significant main effects with genetic treatments (*IGFR-WT vs *IGFR-KO, p=0.004), and no effect from IGF-1 treatment $(\mathrm{p}=0.433)$. ROCK activity was also increased when cultured neurons were inhibited with $0.50 \mu \mathrm{M}$ PPP (Figure 3E, $\mathrm{p}=0.021, \mathrm{n}=10$ ). Supplemental IGF-1 did not significantly alter ROCK activity (Figure 3E, p=0.705). Mechanistically, neither PPP nor genetic reductions in IGF-1R lead to significant increases in ROCK2 protein expression in cultured neurons (Figure 3F p=0.16, Supplemental Figure $2 \mathrm{p}=0.117$ ). mRNA levels of ROCK1, ROCK2, and upstream RhoA were unaffected by $0.50 \mu \mathrm{M}$ PPP (Supplemental Figure 2A, p $>0.05$ ). Neither were total RhoA protein expression, phosphorylated RhoA levels, or the levels of GTP-bound RhoA (Supplemental Figure 2C and Figure 3G, p>0.05), indicating the increase in ROCK activity was not due to robust changes in upstream RhoA activity.

\section{Restoration of Neurite Outgrowth with ROCK Inhibition}

While it is difficult to pharmacologically increase MAPK activity, small molecule inhibitors of ROCK are readily available. Thus, neuron cultures were treated with small molecule ROCK inhibitors, Hydroxyfasudil (HF) and 1-Y27632, in an attempt to restore neurite outgrowth in the presence of the IGFR inhibitor PPP. Both $5 \mu \mathrm{M}$ HF and $1 \mu \mathrm{M} 1-\mathrm{Y} 27632$ significantly increased neurite complexity on their own (Figure 4A-C, $<<0.05, n=6$ ) and prevented decreased complexity with PPP (Figure 4A-C, $\mathrm{p}<0.05$ ). No significant difference was observed in the rescued growth between the either pharmacological ROCK inhibitors.

\section{Spatial Learning and Neuronal Structure Restoration with ROCK Inhibition}

Based on the structural restoration in vitro, we administered $10 \mathrm{mg} / \mathrm{kg}$ HF to male *IGFR-KO mice via osmotic pump for 14 days and assessed whether spatial learning and memory deficits could also be rescued (Figure 5A-B and Supplemental Figure 2). Spatial learning deficits of male *IGFR-KOs in the acquisition phase of the radial arm water maze were not restored after receiving 14-days of HF infusion (Figure 5A-B, p>0.05, n=14). In the reversal phase, HF treated *IGFRKO mice were no different than *IGFR-KO-DMSO controls nor were they statistically different than *IGFR-WT-HF, suggesting a possible partial rescue (Figure 5B). Two-way ANOVA comparisons revealed significant main effects from genetic treatment groups $(p=0.034)$, with no pharmacological drug treatment effect $(p=0.503)$. The total number of errors to find the escape platform in the acquisition and reversal phases showed no significant effects of HF treatment in the *IGFR-WT and/or *IGFR-KO mice (Supplemental Figure 3).

Immediately following behavioral analysis, hippocampal tissue was isolated to assess ROCK activity. A significant increase in ROCK activity was noted in the hippocampi of *IGFR-KODMSO mice compared to *IGFR-WT-DMSO controls (Figure 5C, p=0.02, n=6,7). No statistical difference was noted in *IGFR-KO-HF mice, suggesting bioavailability and accumulation of the ROCK inhibitor in the hippocampus (Figure 5C, $\mathrm{p}>0.05, \mathrm{n}=7$ ). However, Golgi-Cox staining revealed that HF treatment did not increase the number of boutons in the CA1 or dentate gyrus, in 
fact, the bouton number was significantly decreased in the CA1 of mice receiving the ROCK inhibitor (Figure 5D-E, $\mathrm{p}=0.03, \mathrm{n}=8$ ).

\section{DISCUSSION}

The data presented here demonstrate that neuronal IGF-1 signaling is necessary to maintain spatial learning and memory in adult male mice. Previous studies have highlighted how developmental reductions in IGF-1 lead to impairments in neuroglial function, and ultimately impairments in cognitive function. Genetic knockouts of IGF-1 and IGF-1R exhibit reduced brain size decreased myelination, and several behavioral deficits (Beck et al., 1995). The protective effects of IGF-1 on cognitive function are not limited to early-life phenotypes, as the natural decline in circulating IGF-1 in advanced age is associated with increased cognitive deficits in both humans and animal models (Ashpole et al., 2015b; Deijen et al., 2011; Deijen et al., 1998; van Dam et al., 2000). Moreover, the age-related loss of circulating IGF-1 is thought to predispose the aged brain to Alzheimer's Disease and related dementias (Deak and Sonntag, 2012; Doi et al., 2015; Licht et al., 2014; Sonntag et al., 2013; Vidal et al., 2016). Unfortunately, animal models of reduced circulating IGF-1 also exhibit alterations in cardiovascular function, blood-brain barrier integrity, muscle strength, and bone structure due to the extensive anabolic effects of IGF-1R throughout the body (Ashpole et al., 2016; Ashpole et al., 2015a; Ashpole et al., 2017; Farias Quipildor et al., 2019; Kinney et al., 2001; Tarantini et al., 2016a; Tarantini et al., 2016b). This underscores how systemic changes in IGF-1 induces numerous variables that may hamper our interpretation of underlying mechanisms. Thus, we aimed to utilize a mouse model where IGF-1 signaling could be reduced in tissue and cell-specific manner. In addition, we wanted to control the timing of the reduction in IGF-1 signaling to avoid developmental confounds of possible compensatory increases in local IGF-1 production, insulin receptor activity, and insulin-like binding protein prevalence. Therefore, we aimed to delineate the role of neuronal IGF-1 signaling on spatial and working learning and memory in adulthood, after the pubertal surge in IGF-1 has peaked. In the present study, neuronal reduction of IGF-1 in the hippocampus of male mice led to impaired spatial learning, reduced synaptic bouton number, and increased ROCK signaling. While ROCK inhibition rescued neurite outgrowth in vitro, it did not fully restore learning and memory in vivo.

Impairments in spatial learning and memory in the neuronal IGFR-KO mice were accompanied by reductions in hippocampal synapse number, highlighting the possibility that synapse retraction was a potential mechanism underlying cognitive dysfunction. Synaptic pruning and restructuring are markedly increased during puberty, particularly within the hippocampal regions of interest (Anderson et al., 2002). Our goal was to reduce neuronal IGF-1R after this critical developmental window; however, we cannot differentiate whether the reduction in synaptic boutons observed in our knock-out mice is due to increased synaptic pruning or synapse retraction. The possibility of synaptic pruning within our mutant mice is supported by evidence that synaptic pruning is activitydependent, and IGF-1 significantly increases synaptic excitability by modulating NMDA receptor availability, calcium channel function, and inhibitory neurotransmission (Blair et al., 1999; Cao et al., 2011; Gazit et al., 2016; Le Greves et al., 2005; Mardinly et al., 2016). While the reductions in IGF-1R were restricted to neurons, in future studies it would be important to assess microglial 
activation and phagocytosis within the hippocampus of our neuronal IGFR-KO mice to assess whether this mutation increased synaptic pruning. Further analyses of total neuron number and/or newborn neuron incorporation and survival may yield additional information on cellular changes with neuronal IGF-R knockout, especially when one considers previous evidence that IGF-1 promotes survival and differentiation of neurons (Brooker et al., 2000; D'Mello et al., 1997; NietoEstevez et al., 2016). Moreover, a detailed analysis of synapse structure may highlight additional structural differences, as IGF-1 supplementation in aged rats has been shown to increase postsynaptic density and the number of multiple spine boutons(Shi et al., 2005).

Considering the localized restriction of neuronal IGFR deficiency in our study, we focused on hippocampal-dependent behaviors as read-outs of cognitive function. The radial arm water maze showed robust between-group and over-time differences. However, differences were not observed in the novelty recognition assay. The lack of treatment effect within this assay may be influenced by the maintenance of IGF-1 signaling in the other brain regions implicated in novelty seeking behaviors. On the other hand, neither treatment group showed robust novelty discrimination scores above 0.7 . Inclusion of wild-type controls without stereotactic injection of control vectors may be of use to rule out confounds of superior tissue displacement and damage. Additionally, future inducible models of IGFR deletion throughout the entire adult brain may be of interest, as a recent study highlighted that overexpression of IGF-1 within the aged brain had behavior-specific outcomes (Farias Quipildor et al., 2019).

Reductions in MAPK, ERK, and Akt signaling were anticipated with IGF-1 deficiency, as these are the canonical pathways downstream of IGF-1R activation. In addition to reduced MAPK within the hippocampus of our neuronal IGFR-KO mice, we also observed a significant upregulation of RhoA/ROCK activity. As this pathway is well-known to decrease neuronal excitability and induce spine retraction, the observed increase in our neuronal IGFR-KO mice supported our working hypothesis that reduced bouton number may be due to synapse retraction. We utilized a series of in vitro studies to further examine the relationship between IGF-1 and ROCK signaling. Consistent with previous studies, significant increases in neurite extension were observed when RhoA/ROCK signaling was reduced (Chen and Firestein, 2007; Greathouse et al., 2018). Furthermore, ROCK inhibition circumvented the stunted growth associated with IGF-1 inhibition, suggesting a possible route of rescue in our mutant mice. This was particularly interesting considering previous studies have shown small molecule inhibitors of ROCK, including $\mathrm{HF}$, can rescue spatial learning and memory in aged mice and rodent models of neurodegeneration (Guo et al., 2020; Huentelman et al., 2009; Yu et al., 2017). Unfortunately, we did not observe a rescue of synapse number or learning and memory despite our doses of HF falling within the range of these previous studies. In fact, HF mice perplexingly displayed fewer synaptic boutons than controls, without showing signs of spatial learning impairment. It is unclear why bouton number was lower in the HF-treated mice, but it was apparent that ROCK was inhibited in this tissue, which indicates the inhibitor was available when tissues were isolated on the last day of drug administration. When higher-order cognitive flexibility was assessed, the HF knock-outs were not different than wild-type controls or knock-out controls, possibly suggesting a partial rescue. However, this phenotype was not robust nor was it prevalent when other endpoints were examined. 
It is possible that alternative outcomes would have been observed had ROCK been genetically targeted at the same time neuronal IGF-1R was reduced. ROCK inhibitors were not administered until two weeks before behavioral assessment (6 weeks following IGF-1R reduction). Although the 14-day treatment is sufficient to reduce ROCK activity levels to those of controls, it may not be sufficient to allow the reversal of cognitive deficits. Indeed, many of the previous studies with ROCK inhibitors dose animals for two or more months (Busti et al., 2020; Takata et al., 2013; Tonges et al., 2012; Yu et al., 2017). A prophylactic approach may be advantageous to solidify the contributions of ROCK to IGF-1 related deficits.

Sex-specific differences in the response to IGF-1 are increasingly reported (Ashpole et al., 2017; Austad and Fischer, 2016; Bokov et al., 2011; Farias Quipildor et al., 2019; Mao et al., 2018), so it was not surprising to observe impairments limited to male mice. Of note, our experimental design was set for independent assessments of male and female cohorts, rather than crosscomparisons. While sex-specific differences are commonly observed in aged, IGF-1 deficient animals, the mechanism underlying these disparities is not understood. A role for sex hormones has been proposed (Austad, 2019; Austad and Fischer, 2016); however, concrete evidence for this is still lacking. Additional studies focused on the mechanism underlying the sex-dependence of the effect of IGF-1 in the brain are warranted, and would reveal critical information needed across the fields of developmental biology and geroscience.

Overall, we observe significant impairments in spatial learning and memory when IGF-1 signaling is reduced in neurons of adult male mice. These results provide potential mechanistic insights underlying learning and memory impairments in advanced age when IGF-1 levels are significantly reduced. Considering the therapeutic limitations of exogenous IGF-1 administration in the elderly, the new focus should now turn downstream of neuronal IGF-1R to identify potential therapeutics that could rescue or prevent the loss of cognitive function.

\section{FIGURE LEGENDS}

Figure 1: Adult IGF-1R Signaling in Neurons Regulates Spatial Learning and Memory in Male Mice. (A) Experimental design and timeline of hippocampal neuron-specific deletion of IGFR (*IGFR-KO), behavioral testing, and ex vivo analysis. Image made with biorender.com (B) Hippocampal mRNA expression of IGF-1R, IGF-1, growth hormone receptor (GHR), and insulin receptor (InsR) in *IGFR-KO mice, normalized to control *IGFR-WT (mean +/- SEM, n=9). Geometric means of HPRT and B2M were utilized as housekeeper genes. The asterisk indicates a significant difference of each gene vs*IGFR-WT control $\left({ }^{*} \mathrm{p}<0.05\right.$, t-test). (C-F) Assessment of spatial learning and memory in male *IGFR-WT and *IGFR-KO mice within the radial arm water maze. Average pathlength traveled per trial by male mice during the acquisition phase (C) and reversal phase (D) of the radial arm water maze. Average number of errors per trial in the acquisition phase (E) and reversal phase (F) of the radial arm water maze. The \# denotes $\mathrm{p}<0.05$ across treatment groups in the acquisition phase (mean +/- SEM, n=10,11, two-wayANOVA repeated measures). The asterisk denotes $\mathrm{p}<0.05$ vs *IGFR-WT in the reversal phase (t-test). (G) Average pathlength of male *IGFR-WT and *IGFR-KO mice in each of the five phases of the novel object/relocation task ( $\mathrm{p}>0.05$, two-way ANOVA repeated measures). (H) Average discrimination between the object relocated during the novel location trial ( $>>0.05$, t-test). (I) 
Average discrimination between the new and old object during the novel object ( $\mathrm{p}>0.05$, $\mathrm{t}$-test). All novel object data presented as mean +/- SEM, $n=11,10$.

Figure 2: Alterations in hippocampal neuron structure with neuronal IGF-1R deficiency. (A) Representative images of synaptic boutons on dendrites in CA1 of *IGFR-WT and *IGFR-KO male mice. (B) The average number of boutons across $20 \mu \mathrm{m}$ of a dendrite within the CA1 (minimum 2 dendrites per mouse, mouse $n=9,12$ ). The asterisk denotes $p<0.05$, t-test. (C) Representative images of cultured hippocampal neurons treated with increasing concentrations of IGF-1R inhibitor, PPP. (D) Sholl analysis tracing quantification of the average number of crossings every 10 microns from the soma with increasing PPP, 24 hours following PPP administration. (E) The average area under the curve from (D); ( $n=6,8$ fields). All data are represented as mean $+/-$ SEM and an asterisk denotes $\mathrm{p}<0.05$ vs the DMSO vehicle control (One-way ANOVA, post-hoc Dunnett's test). PPP picropodophyllin toxin.

Figure 3: Alterations in MAPK and ROCK signaling with neuronal IGFR deficiency. (A) Left: Representative image of MAPK western blots, Right: Average ratio of phosphorylated MAPK to total MAPK in male *IGFR-WT and *IGFR-KO hippocampal lysates. The asterisk denotes $\mathrm{p}<0.05$, t-test. (B) Average ROCK activity in male *IGFR-WT and *IGFR-KO hippocampal lysates. The asterisk denotes $\mathrm{p}<0.05$, t-test. (C) Left: Representative image of western blots Right: Average ratio of phosphocofilin to total cofilin in male*IGFR-WT and *IGFR-KO hippocampal lysates. (D) ROCK activity in cultured igfrf/f neurons treated 48 hours after treatment with AAV9-Syn-Cre (or AAV9-Syn-GFP), with and without 100nM supplemented IGF-1 in cell culture. The asterisk indicates the significant main effect of WT vs KO, with no difference in IGF-1 treatment condition (two-way ANOVA posthoc Bonferonni, genetic treatment, and IGF-1 treatment as factors). (E) Average ROCK activity levels in cultured neurons treated with $0.5 \mu \mathrm{M}$ PPP, 100nM DMSO vehicle. The asterisk indicates a significant difference compared to DMSO control (One-way ANOVA, post-hoc Dunnett's vs control). Average ROCK2 protein expression (Left: representative western blots, Right: quantification) (F) and GTP-bound RhoA levels (G) in cultured neurons treated with IGFR inhibitor $0.5 \mu \mathrm{M}$ PPP. All data is represented by mean $+/$ - SEM.

Figure 4: The pharmacological inhibition and restoration of neurite outgrowth with IGF-1R and ROCK inhibitors. (A) Representative images of neuronal growth with PPP (IGF-1R inhibitor) and ROCK inhibitors (HF and Y-27632). (B) Sholl analysis of the average number of neuronal crossings every $10 \mu \mathrm{m}$ from the soma in neurons treated with DMSO vehicle, $0.5 \mu \mathrm{M}$ PPP, $5 \mu \mathrm{M}$ HF, and $1 \mu \mathrm{M}$ Y-27632 treatments. (C) The average area under the curves in $\mathbf{B}$ (6 neurons per coverslip, $n=3,4$ coverslips). Asterisk denotes $\mathrm{p}<0.05$ for knock-out effect, (Two-way ANOVA, post-hoc Bonferonni multiple comparisons). Abbreviations: N.D. no difference, PPP picropodophyllin toxin, HF Hydroxyfasudil.

Figure 5: Partial restoration of spatial learning and memory and synaptic bouton number with HF infusion. Mice were implanted with 14-day releasing mini-osmotic pumps loaded with $10 \mathrm{mg} / \mathrm{kg}$ HF or DMSO vehicle. (A) Average total pathlength traveled per trial on each day of the acquisition phase of the radial arm water maze $(n=14)$. (B) Average pathlength traveled of four 
treatment groups during the reversal phase of the radial arm water maze $(n=14)$. Asterisk denotes $\mathrm{p}<0.05$ for WT vs KO, while N.D. denotes no statistical difference ( $\mathrm{p}>0.05$, two-way ANOVA, post-hoc Bonferonni multiple comparisons) (C) Average ROCK activity within the hippocampal lysates of *IGFR-WT and *IGFR-KO mice treated with DMSO vehicle and HF $(n=6,7)$. Average number of synaptic boutons across $20 \mu \mathrm{m}$ of dendrites in the CA1 (D) and DG $(\mathbf{E})(\mathrm{n}=7,10)$. Asterisk denotes $\mathrm{p}<0.05$ to respective control (t-test). All data are expressed as mean $+/-\mathrm{SEM}$.

Acknowledgments: The authors would like to thank the animal care staff at both the University of Oklahoma Health Sciences Center and the University of Mississippi.

Funding: This research was funded by the National Institutes of Health [F32-AG048727, R15AG059142-01]; and the Southern Research Education Board Fellowship.

\section{REFERENCES}

Anderson, M.F., Aberg, M.A., Nilsson, M., Eriksson, P.S., 2002. Insulin-like growth factor-I and neurogenesis in the adult mammalian brain. Brain Res Dev Brain Res 134, 115-122.

Arwert, L.I., Veltman, D.J., Deijen, J.B., van Dam, P.S., Drent, M.L., 2006. Effects of growth hormone substitution therapy on cognitive functioning in growth hormone deficient patients: a functional MRI study. Neuroendocrinology 83, 12-19.

Ashpole, N.M., Herron, J.C., Estep, P.N., Logan, S., Hodges, E.L., Yabluchanskiy, A., Humphrey, M.B., Sonntag, W.E., 2016. Differential effects of IGF-1 deficiency during the life span on structural and biomechanical properties in the tibia of aged mice. Age 38, 38.

Ashpole, N.M., Herron, J.C., Mitschelen, M.C., Farley, J.A., Logan, S., Yan, H., Ungvari, Z., Hodges, E.L., Csiszar, A., Ikeno, Y., Humphrey, M.B., Sonntag, W.E., 2015a. IGF-1 Regulates Vertebral Bone Aging Through Sex-Specific and Time-Dependent Mechanisms. Journal of bone and mineral research : the official journal of the American Society for Bone and Mineral Research.

Ashpole, N.M., Hudmon, A., 2011. Excitotoxic neuroprotection and vulnerability with CaMKII inhibition. Mol Cell Neurosci 46, 720-730.

Ashpole, N.M., Logan, S., Yabluchanskiy, A., Mitschelen, M.C., Yan, H., Farley, J.A., Hodges, E.L., Ungvari, Z., Csiszar, A., Chen, S., Georgescu, C., Hubbard, G.B., Ikeno, Y., Sonntag, W.E., 2017. IGF-1 has sexually dimorphic, pleiotropic, and time-dependent effects on healthspan, pathology, and lifespan. Geroscience 39, 129-145.

Ashpole, N.M., Sanders, J.E., Hodges, E.L., Yan, H., Sonntag, W.E., 2015b. Growth hormone, insulinlike growth factor-1 and the aging brain. Experimental gerontology 68, 76-81.

Austad, S.N., 2019. Sex differences in health and aging: a dialog between the brain and gonad? Geroscience 41, 267-273.

Austad, S.N., Fischer, K.E., 2016. Sex Differences in Lifespan. Cell metabolism 23, 1022-1033.

Barbash, S., Hanin, G., Soreq, H., 2013. Stereotactic injection of microRNA-expressing lentiviruses to the mouse hippocampus ca1 region and assessment of the behavioral outcome. J Vis Exp, e50170.

Beck, K.D., Powell-Braxton, L., Widmer, H.R., Valverde, J., Hefti, F., 1995. Igf1 gene disruption results in reduced brain size, CNS hypomyelination, and loss of hippocampal granule and striatal parvalbumincontaining neurons. Neuron 14, 717-730.

Bito, H., Furuyashiki, T., Ishihara, H., Shibasaki, Y., Ohashi, K., Mizuno, K., Maekawa, M., Ishizaki, T., Narumiya, S., 2000. A critical role for a Rho-associated kinase, p160ROCK, in determining axon outgrowth in mammalian CNS neurons. Neuron 26, 431-441.

Blair, L.A., Bence-Hanulec, K.K., Mehta, S., Franke, T., Kaplan, D., Marshall, J., 1999. Akt-dependent potentiation of $\mathrm{L}$ channels by insulin-like growth factor-1 is required for neuronal survival. J Neurosci 19, 1940-1951. 
Bokov, A.F., Garg, N., Ikeno, Y., Thakur, S., Musi, N., DeFronzo, R.A., Zhang, N., Erickson, R.C., Gelfond, J., Hubbard, G.B., Adamo, M.L., Richardson, A., 2011. Does Reduced IGF-1R Signaling in Igf1r(+/-) Mice Alter Aging? Plos One 6.

Brooker, G.J., Kalloniatis, M., Russo, V.C., Murphy, M., Werther, G.A., Bartlett, P.F., 2000. Endogenous IGF-1 regulates the neuronal differentiation of adult stem cells. Journal of neuroscience research 59, 332341.

Busti, I., Allegra, M., Spalletti, C., Panzi, C., Restani, L., Billuart, P., Caleo, M., 2020. ROCK/PKA Inhibition Rescues Hippocampal Hyperexcitability and GABAergic Neuron Alterations in a

Oligophrenin-1 Knock-Out Mouse Model of X-Linked Intellectual Disability. J Neurosci 40, 2776-2788.

Cao, P., Maximov, A., Sudhof, T.C., 2011. Activity-dependent IGF-1 exocytosis is controlled by the $\mathrm{Ca}(2+)$-sensor synaptotagmin-10. Cell 145, 300-311.

Chen, H., Firestein, B.L., 2007. RhoA regulates dendrite branching in hippocampal neurons by decreasing cypin protein levels. J Neurosci 27, 8378-8386.

Cheng, C.M., Mervis, R.F., Niu, S.L., Salem, N., Jr., Witters, L.A., Tseng, V., Reinhardt, R., Bondy, C.A., 2003. Insulin-like growth factor 1 is essential for normal dendritic growth. Journal of neuroscience research 73, 1-9.

D'Mello, S.R., Borodezt, K., Soltoff, S.P., 1997. Insulin-like growth factor and potassium depolarization maintain neuronal survival by distinct pathways: possible involvement of PI 3-kinase in IGF-1 signaling. J Neurosci 17, 1548-1560.

Dash, P.K., Orsi, S.A., Moody, M., Moore, A.N., 2004. A role for hippocampal Rho-ROCK pathway in long-term spatial memory. Biochem Biophys Res Commun 322, 893-898.

Deak, F., Sonntag, W.E., 2012. Aging, synaptic dysfunction, and insulin-like growth factor (IGF)-1. The journals of gerontology. Series A, Biological sciences and medical sciences 67, 611-625.

Deijen, J.B., Arwert, L.I., Drent, M.L., 2011. The GH/IGF-I Axis and Cognitive Changes across a 4-Year Period in Healthy Adults. ISRN endocrinology 2011, 249421.

Deijen, J.B., de Boer, H., van der Veen, E.A., 1998. Cognitive changes during growth hormone replacement in adult men. Psychoneuroendocrinology 23, 45-55.

Doi, T., Shimada, H., Makizako, H., Tsutsumimoto, K., Hotta, R., Nakakubo, S., Suzuki, T., 2015. Association of insulin-like growth factor-1 with mild cognitive impairment and slow gait speed. Neurobiol Aging 36, 942-947.

Farias Quipildor, G.E., Mao, K., Hu, Z., Novaj, A., Cui, M.H., Gulinello, M., Branch, C.A., Gubbi, S., Patel, K., Moellering, D.R., Tarantini, S., Kiss, T., Yabluchanskiy, A., Ungvari, Z., Sonntag, W.E., Huffman, D.M., 2019. Central IGF-1 protects against features of cognitive and sensorimotor decline with aging in male mice. Geroscience 41, 185-208.

Florini, J.R., Prinz, P.N., Vitiello, M.V., Hintz, R.L., 1985. Somatomedin-C levels in healthy young and old men: relationship to peak and 24-hour integrated levels of growth hormone. J Gerontol 40, 2-7. Frater, J., Lie, D., Bartlett, P., McGrath, J.J., 2018. Insulin-like Growth Factor 1 (IGF-1) as a marker of cognitive decline in normal ageing: A review. Ageing Res Rev 42, 14-27.

Gazit, N., Vertkin, I., Shapira, I., Helm, M., Slomowitz, E., Sheiba, M., Mor, Y., Rizzoli, S., Slutsky, I., 2016. IGF-1 Receptor Differentially Regulates Spontaneous and Evoked Transmission via Mitochondria at Hippocampal Synapses. Neuron 89, 583-597.

Glasper, E.R., Llorens-Martin, M.V., Leuner, B., Gould, E., Trejo, J.L., 2010. Blockade of insulin-like growth factor-I has complex effects on structural plasticity in the hippocampus. Hippocampus 20, 706712.

Greathouse, K.M., Boros, B.D., Deslauriers, J.F., Henderson, B.W., Curtis, K.A., Gentry, E.G., Herskowitz, J.H., 2018. Distinct and complementary functions of rho kinase isoforms ROCK1 and ROCK2 in prefrontal cortex structural plasticity. Brain Struct Funct 223, 4227-4241.

Grinberg, Y.Y., Dibbern, M.E., Levasseur, V.A., Kraig, R.P., 2013. Insulin-like growth factor-1 abrogates microglial oxidative stress and TNF-alpha responses to spreading depression. J Neurochem 126, 662-672. Guo, M.F., Zhang, H.Y., Li, Y.H., Gu, Q.F., Wei, W.Y., Wang, Y.Y., Zhang, X.J., Liu, X.Q., Song, L.J., Chai, Z., Yu, J.Z., Ma, C.G., 2020. Fasudil inhibits the activation of microglia and astrocytes of 
transgenic Alzheimer's disease mice via the downregulation of TLR4/Myd88/NF-kappaB pathway. J Neuroimmunol 346, 577284.

Hirose, M., Ishizaki, T., Watanabe, N., Uehata, M., Kranenburg, O., Moolenaar, W.H., Matsumura, F., Maekawa, M., Bito, H., Narumiya, S., 1998. Molecular dissection of the Rho-associated protein kinase (p160ROCK)-regulated neurite remodeling in neuroblastoma N1E-115 cells. The Journal of cell biology 141, 1625-1636.

Huentelman, M.J., Stephan, D.A., Talboom, J., Corneveaux, J.J., Reiman, D.M., Gerber, J.D., Barnes, C.A., Alexander, G.E., Reiman, E.M., Bimonte-Nelson, H.A., 2009. Peripheral delivery of a ROCK inhibitor improves learning and working memory. Behav Neurosci 123, 218-223.

Johanson, A.J., Blizzard, R.M., 1981. Low somatomedin-C levels in older men rise in response to growth hormone administration. The Johns Hopkins medical journal 149, 115-117.

Kinney, B.A., Meliska, C.J., Steger, R.W., Bartke, A., 2001. Evidence that Ames dwarf mice age differently from their normal siblings in behavioral and learning and memory parameters. Horm Behav 39, 277-284.

Lamprecht, R., Farb, C.R., LeDoux, J.E., 2002. Fear memory formation involves p190 RhoGAP and ROCK proteins through a GRB2-mediated complex. Neuron 36, 727-738.

Le Greves, M., Le Greves, P., Nyberg, F., 2005. Age-related effects of IGF-1 on the NMDA-, GH- and IGF-1-receptor mRNA transcripts in the rat hippocampus. Brain Res Bull 65, 369-374.

Licht, C.M., van Turenhout, L.C., Deijen, J.B., Koppes, L.L., van Mechelen, W., Twisk, J.W., Drent, M.L., 2014. The Association between IGF-1 Polymorphisms, IGF-1 Serum Levels, and Cognitive Functions in Healthy Adults: The Amsterdam Growth and Health Longitudinal Study. Int J Endocrinol 2014, 181327.

Logan, S., Pharaoh, G.A., Marlin, M.C., Masser, D.R., Matsuzaki, S., Wronowski, B., Yeganeh, A., Parks, E.E., Premkumar, P., Farley, J.A., Owen, D.B., Humphries, K.M., Kinter, M., Freeman, W.M., Szweda, L.I., Van Remmen, H., Sonntag, W.E., 2018. Insulin-like growth factor receptor signaling regulates working memory, mitochondrial metabolism, and amyloid-beta uptake in astrocytes. Mol Metab 9, 141-155.

Lopez-Otin, C., Blasco, M.A., Partridge, L., Serrano, M., Kroemer, G., 2013. The hallmarks of aging. Cell 153, 1194-1217.

Maher, F.O., Clarke, R.M., Kelly, A., Nally, R.E., Lynch, M.A., 2006. Interaction between interferon gamma and insulin-like growth factor-1 in hippocampus impacts on the ability of rats to sustain long-term potentiation. J Neurochem 96, 1560-1571.

Mao, K., Quipildor, G.F., Tabrizian, T., Novaj, A., Guan, F., Walters, R.O., Delahaye, F., Hubbard, G.B., Ikeno, Y., Ejima, K., Li, P., Allison, D.B., Salimi-Moosavi, H., Beltran, P.J., Cohen, P., Barzilai, N., Huffman, D.M., 2018. Late-life targeting of the IGF-1 receptor improves healthspan and lifespan in female mice. Nat Commun 9, 2394.

Mardinly, A.R., Spiegel, I., Patrizi, A., Centofante, E., Bazinet, J.E., Tzeng, C.P., Mandel-Brehm, C., Harmin, D.A., Adesnik, H., Fagiolini, M., Greenberg, M.E., 2016. Sensory experience regulates cortical inhibition by inducing IGF1 in VIP neurons. Nature 531, 371-375.

Markowska, A.L., Mooney, M., Sonntag, W.E., 1998. Insulin-like growth factor-1 ameliorates age-related behavioral deficits. Neuroscience 87, 559-569.

Meziane, H., Khelfaoui, M., Morello, N., Hiba, B., Calcagno, E., Reibel-Foisset, S., Selloum, M., Chelly, J., Humeau, Y., Riet, F., Zanni, G., Herault, Y., Bienvenu, T., Giustetto, M., Billuart, P., 2016. Fasudil treatment in adult reverses behavioural changes and brain ventricular enlargement in Oligophrenin-1 mouse model of intellectual disability. Hum Mol Genet 25, 2314-2323.

Nieto-Estevez, V., Oueslati-Morales, C.O., Li, L., Pickel, J., Morales, A.V., Vicario-Abejon, C., 2016. Brain Insulin-Like Growth Factor-I Directs the Transition from Stem Cells to Mature Neurons During Postnatal/Adult Hippocampal Neurogenesis. Stem Cells 34, 2194-2209.

Park, S.E., Dantzer, R., Kelley, K.W., McCusker, R.H., 2011. Central administration of insulin-like growth factor-I decreases depressive-like behavior and brain cytokine expression in mice. $\mathrm{J}$

Neuroinflammation 8, 12. 
Prabhu, D., Khan, S.M., Blackburn, K., Marshall, J.P., Ashpole, N.M., 2019. Loss of insulin-like growth factor-1 signaling in astrocytes disrupts glutamate handling. J Neurochem.

Rudman, D., Kutner, M.H., Rogers, C.M., Lubin, M.F., Fleming, G.A., Bain, R.P., 1981. Impaired growth hormone secretion in the adult population: relation to age and adiposity. J Clin Invest 67, 13611369.

Scolnick, J.A., Cui, K., Duggan, C.D., Xuan, S., Yuan, X.B., Efstratiadis, A., Ngai, J., 2008. Role of IGF signaling in olfactory sensory map formation and axon guidance. Neuron 57, 847-857.

Shanmugalingam, T., Bosco, C., Ridley, A.J., Van Hemelrijck, M., 2016. Is there a role for IGF-1 in the development of second primary cancers? Cancer Med 5, 3353-3367.

Shi, L., Linville, M.C., Tucker, E.W., Sonntag, W.E., Brunso-Bechtold, J.K., 2005. Differential effects of aging and insulin-like growth factor-1 on synapses in CA1 of rat hippocampus. Cereb Cortex 15, 571577.

Sonntag, W.E., Carter, C.S., Ikeno, Y., Ekenstedt, K., Carlson, C.S., Loeser, R.F., Chakrabarty, S., Lee, S., Bennett, C., Ingram, R., Moore, T., Ramsey, M., 2005a. Adult-onset growth hormone and insulin-like growth factor I deficiency reduces neoplastic disease, modifies age-related pathology, and increases life span. Endocrinology 146, 2920-2932.

Sonntag, W.E., Deak, F., Ashpole, N., Toth, P., Csiszar, A., Freeman, W., Ungvari, Z., 2013. Insulin-like growth factor-1 in CNS and cerebrovascular aging. Front Aging Neurosci 5, 27.

Sonntag, W.E., Ramsey, M., Carter, C.S., 2005b. Growth hormone and insulin-like growth factor-1 (IGF1) and their influence on cognitive aging. Ageing Res Rev 4, 195-212.

Stoica, L., Ahmed, S.S., Gao, G., Sena-Esteves, M., 2013. Gene transfer to the CNS using recombinant adeno-associated virus. Curr Protoc Microbiol Chapter 14, Unit14D 15.

Sunico, C.R., Gonzalez-Forero, D., Dominguez, G., Garcia-Verdugo, J.M., Moreno-Lopez, B., 2010. Nitric oxide induces pathological synapse loss by a protein kinase G-, Rho kinase-dependent mechanism preceded by myosin light chain phosphorylation. J Neurosci 30, 973-984.

Takata, M., Tanaka, H., Kimura, M., Nagahara, Y., Tanaka, K., Kawasaki, K., Seto, M., Tsuruma, K., Shimazawa, M., Hara, H., 2013. Fasudil, a rho kinase inhibitor, limits motor neuron loss in experimental models of amyotrophic lateral sclerosis. Br J Pharmacol 170, 341-351.

Tarantini, S., Giles, C.B., Wren, J.D., Ashpole, N.M., Valcarcel-Ares, M.N., Wei, J.Y., Sonntag, W.E., Ungvari, Z., Csiszar, A., 2016a. IGF-1 deficiency in a critical period early in life influences the vascular aging phenotype in mice by altering miRNA-mediated post-transcriptional gene regulation: implications for the developmental origins of health and disease hypothesis. Age 38, 239-258.

Tarantini, S., Tucsek, Z., Valcarcel-Ares, M.N., Toth, P., Gautam, T., Giles, C.B., Ballabh, P., Wei, J.Y., Wren, J.D., Ashpole, N.M., Sonntag, W.E., Ungvari, Z., Csiszar, A., 2016b. Circulating IGF-1 deficiency exacerbates hypertension-induced microvascular rarefaction in the mouse hippocampus and retrosplenial cortex: implications for cerebromicrovascular and brain aging. Age 38, 273-289.

Tonges, L., Frank, T., Tatenhorst, L., Saal, K.A., Koch, J.C., Szego, E.M., Bahr, M., Weishaupt, J.H., Lingor, P., 2012. Inhibition of rho kinase enhances survival of dopaminergic neurons and attenuates axonal loss in a mouse model of Parkinson's disease. Brain 135, 3355-3370.

Trejo, J.L., Piriz, J., Llorens-Martin, M.V., Fernandez, A.M., Bolos, M., LeRoith, D., Nunez, A., TorresAleman, I., 2007. Central actions of liver-derived insulin-like growth factor I underlying its pro-cognitive effects. Molecular psychiatry 12, 1118-1128.

van Dam, P.S., Aleman, A., de Vries, W.R., Deijen, J.B., van der Veen, E.A., de Haan, E.H., Koppeschaar, H.P., 2000. Growth hormone, insulin-like growth factor I and cognitive function in adults. Growth hormone \& IGF research : official journal of the Growth Hormone Research Society and the International IGF Research Society 10 Suppl B, S69-73.

VanGuilder Starkey, H.D., Sonntag, W.E., Freeman, W.M., 2013. Increased hippocampal NgR1 signaling machinery in aged rats with deficits of spatial cognition. Eur J Neurosci 37, 1643-1658.

Vidal, J.S., Hanon, O., Funalot, B., Brunel, N., Viollet, C., Rigaud, A.S., Seux, M.L., le-Bouc, Y., Epelbaum, J., Duron, E., 2016. Low Serum Insulin-Like Growth Factor-I Predicts Cognitive Decline in Alzheimer's Disease. J Alzheimers Dis 52, 641-649. 
bioRxiv preprint doi: https://doi.org/10.1101/2021.08.08.455596; this version posted August 9, 2021. The copyright holder for this preprint (which was not certified by peer review) is the author/funder. All rights reserved. No reuse allowed without permission.

Yu, J.Z., Li, Y.H., Liu, C.Y., Wang, Q., Gu, Q.F., Wang, H.Q., Zhang, G.X., Xiao, B.G., Ma, C.G., 2017. Multitarget Therapeutic Effect of Fasudil in APP/PS1transgenic Mice. CNS Neurol Disord Drug Targets 16, 199-209. 
FIGURE 1:

A.

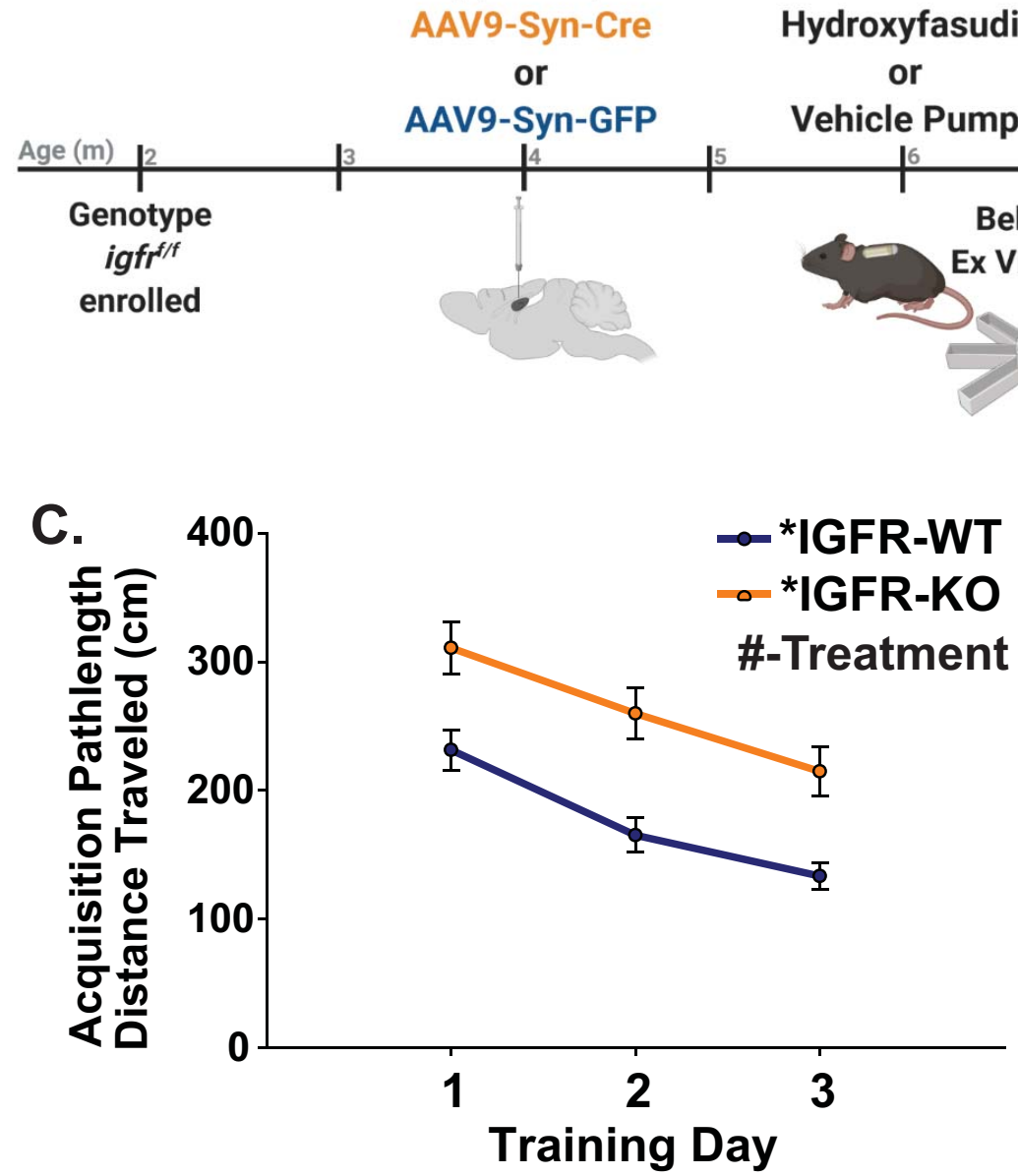

E.

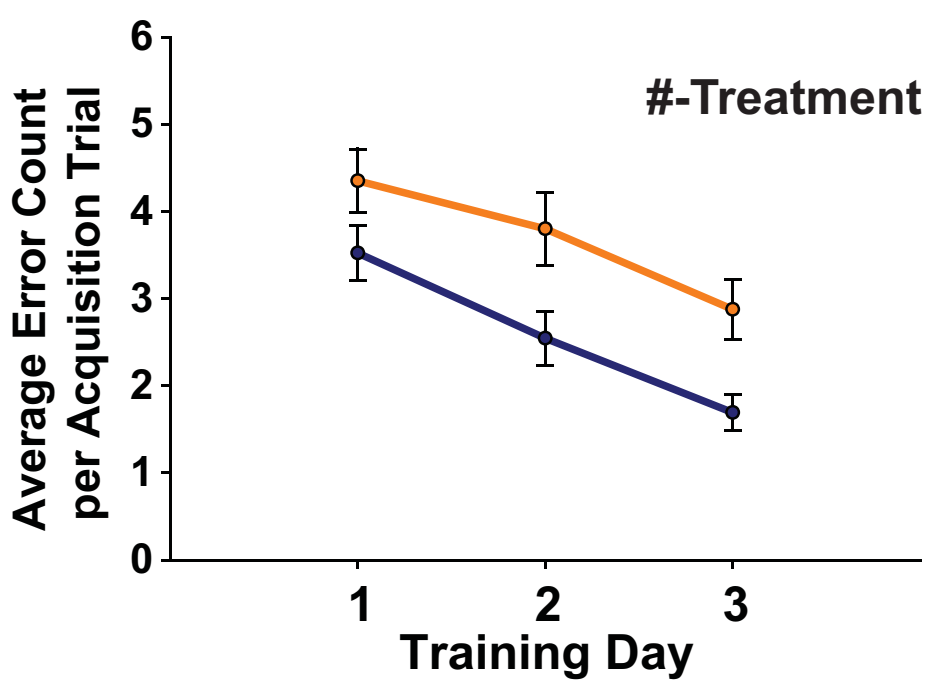

$\begin{array}{cc}\text { Hippocampal } & \text { Subcutaneous } \\ \text { AAV9-Syn-Cre } & \text { Hydroxyfasudil } \\ \text { or } & \text { or }\end{array}$ Ex Vivo Analysis

igfr $^{f / f}$

C.

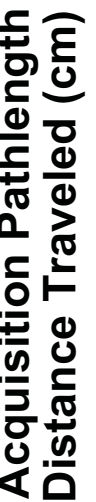

$\ldots$ *IGFR-WT

\#-Treatment

D.
B.

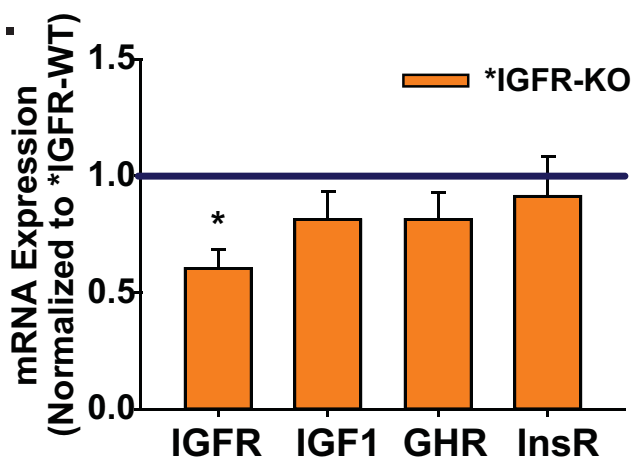

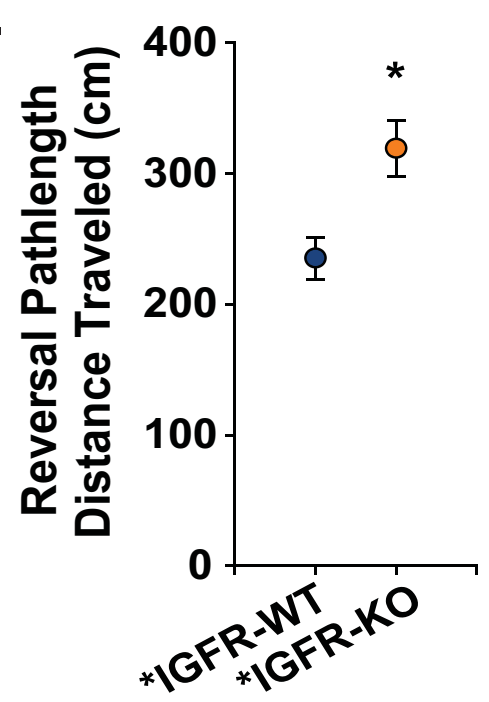

F.

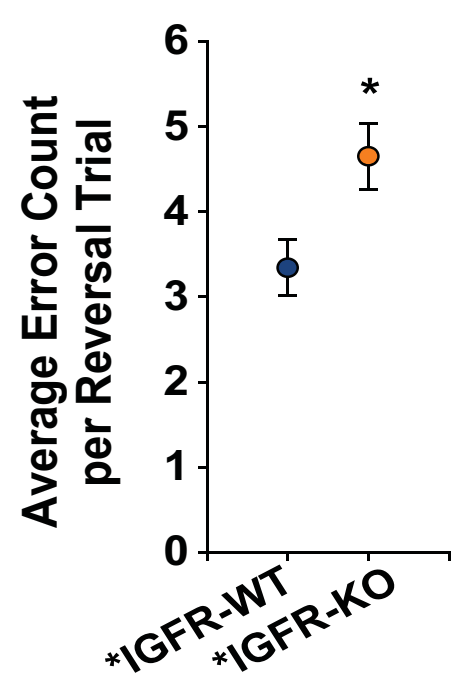

G

H.
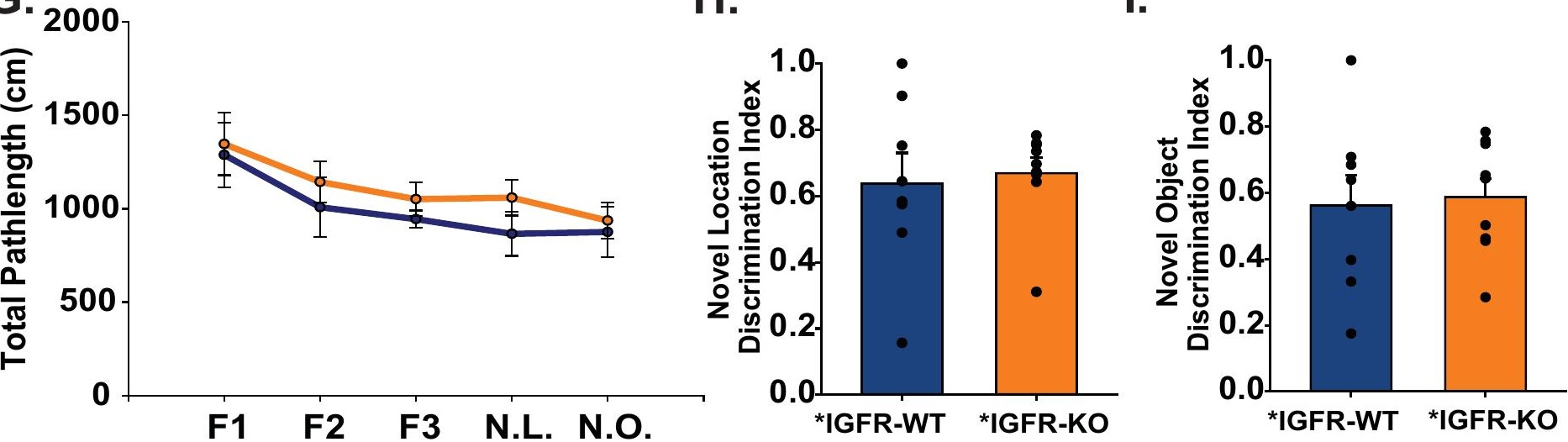
FIGURE 2:

A.

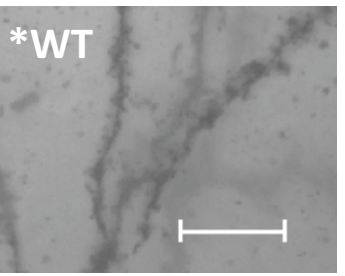

*KO

$10 \mu \mathrm{m}$
B.

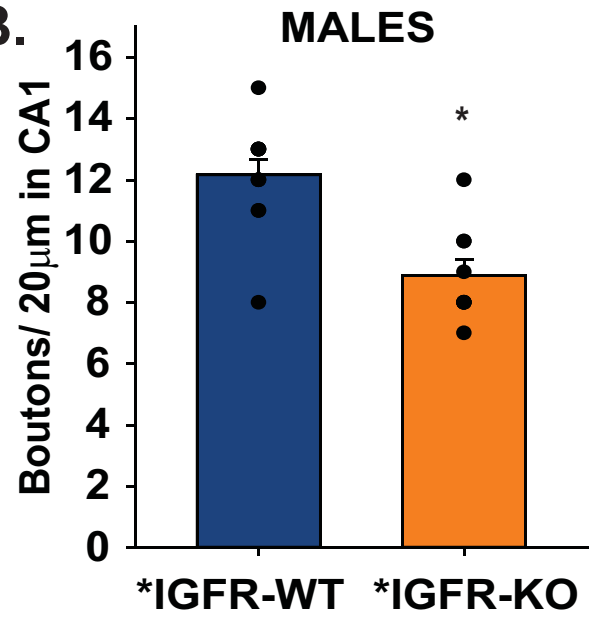

C.

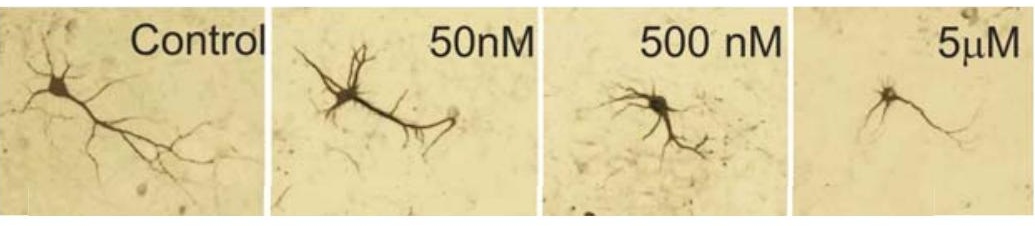

D.

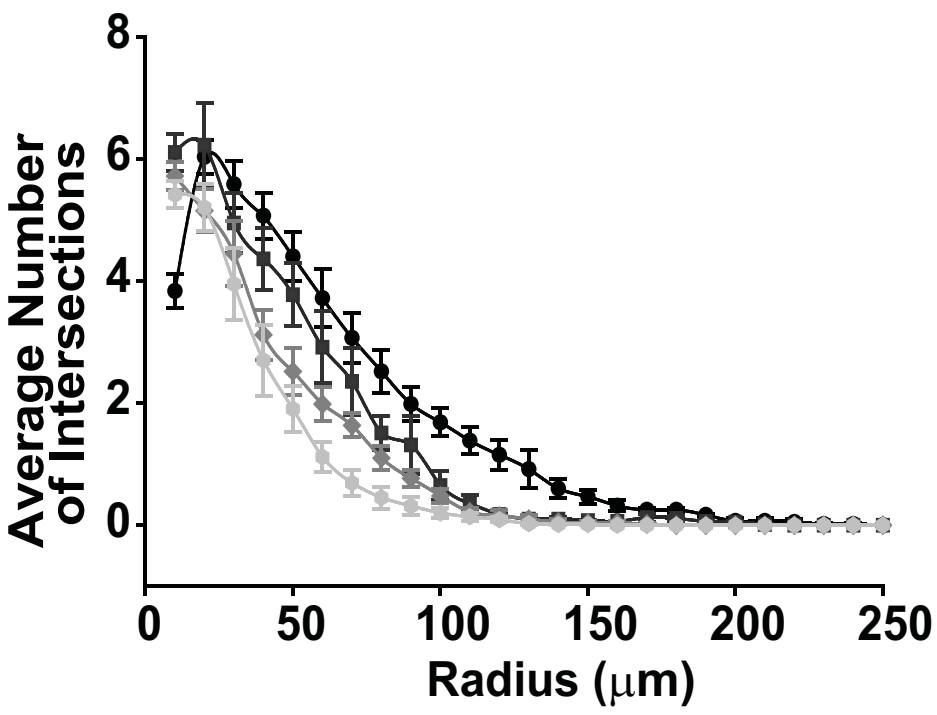

E.

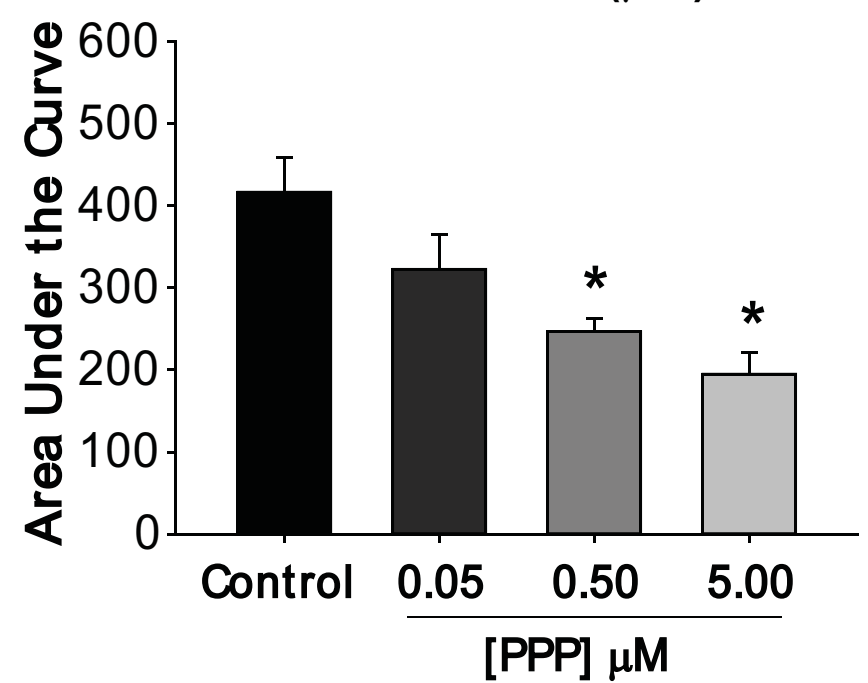


FIGURE 3:

A.

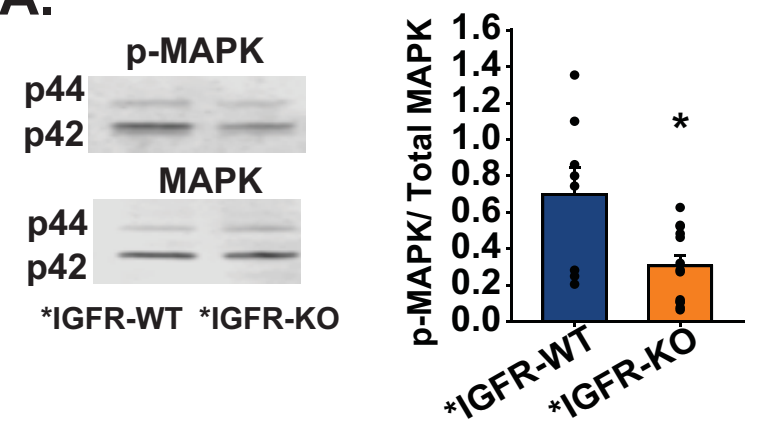

C.

p-Cofilin

$18 \mathrm{kDa}$

\section{Cofilin}

$18 \mathrm{kDa}$

*IGFR-WT *IGFR-KO
B. 0.05 Hippocampal Tissue

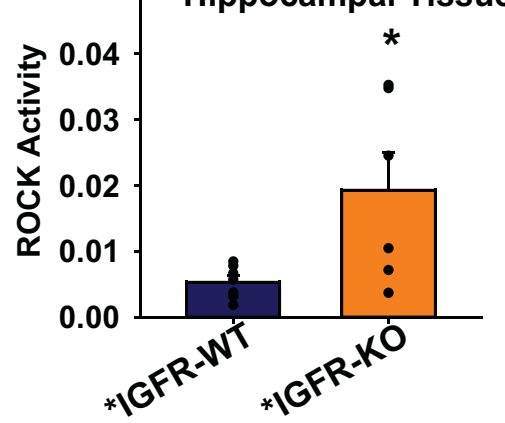

D.

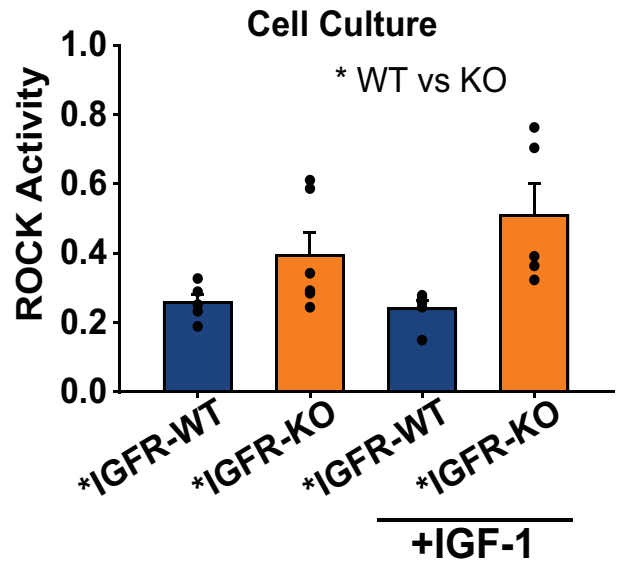

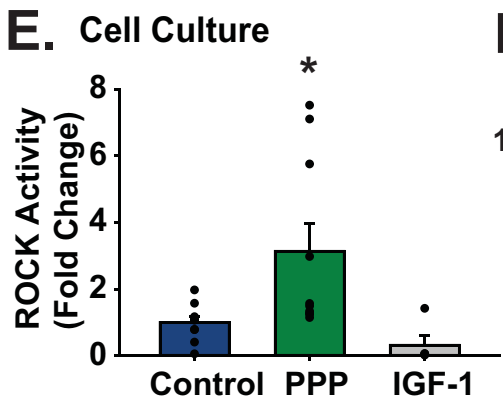

E.

$150 \mathrm{kDa}$

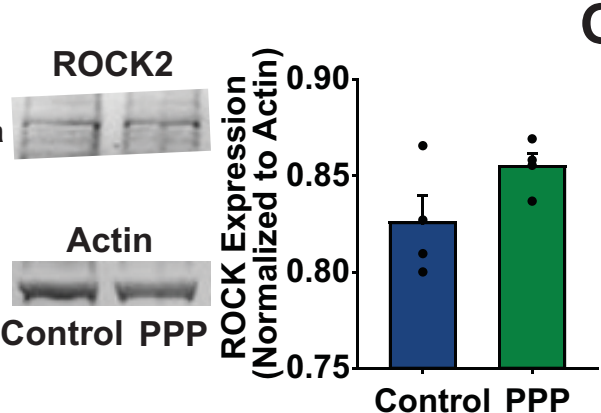

G.

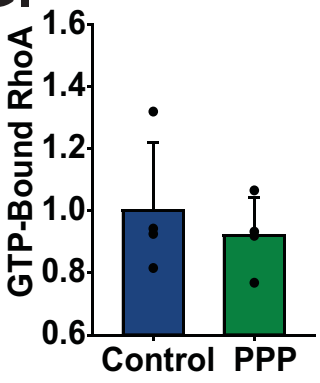


FIGURE 4
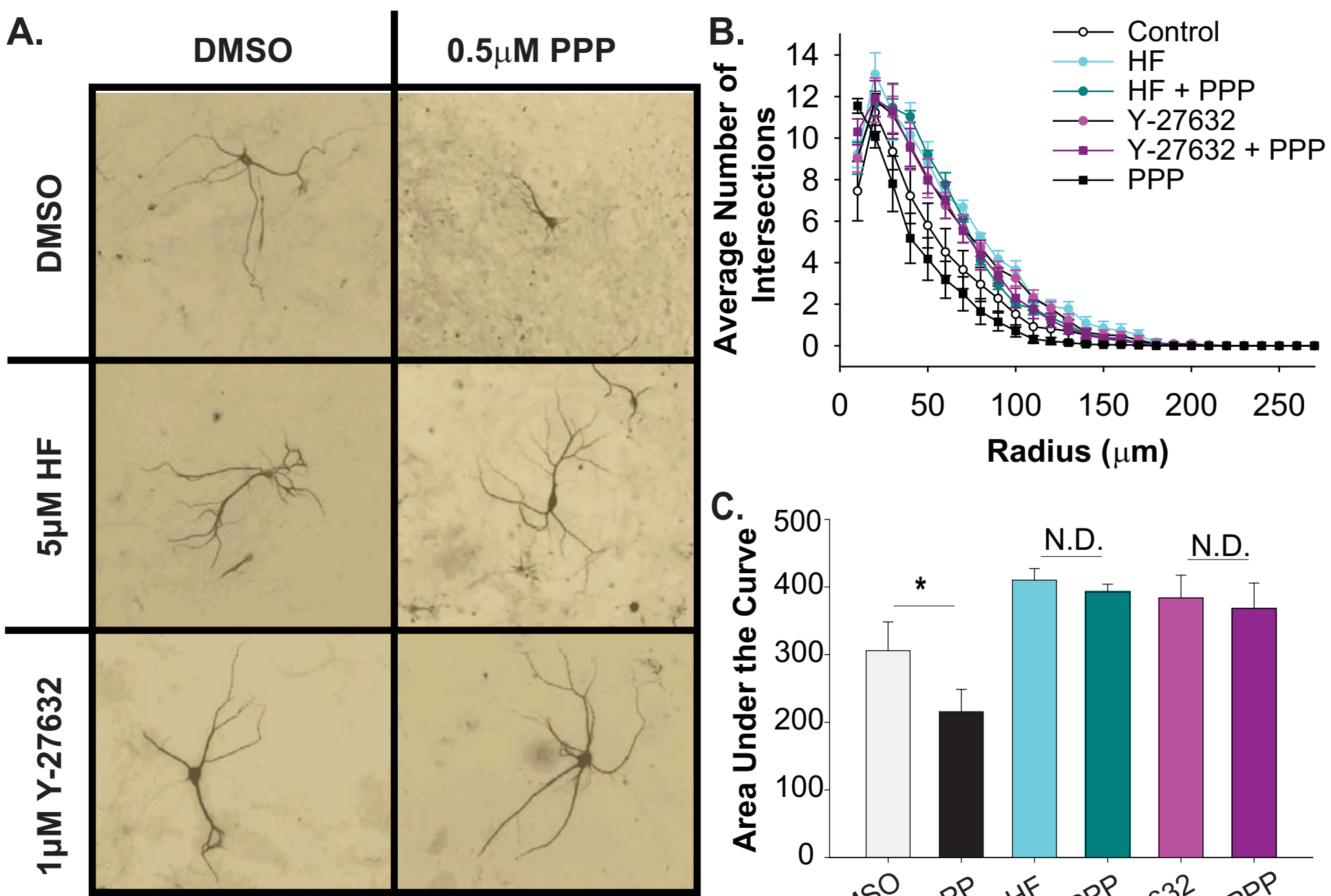

C.

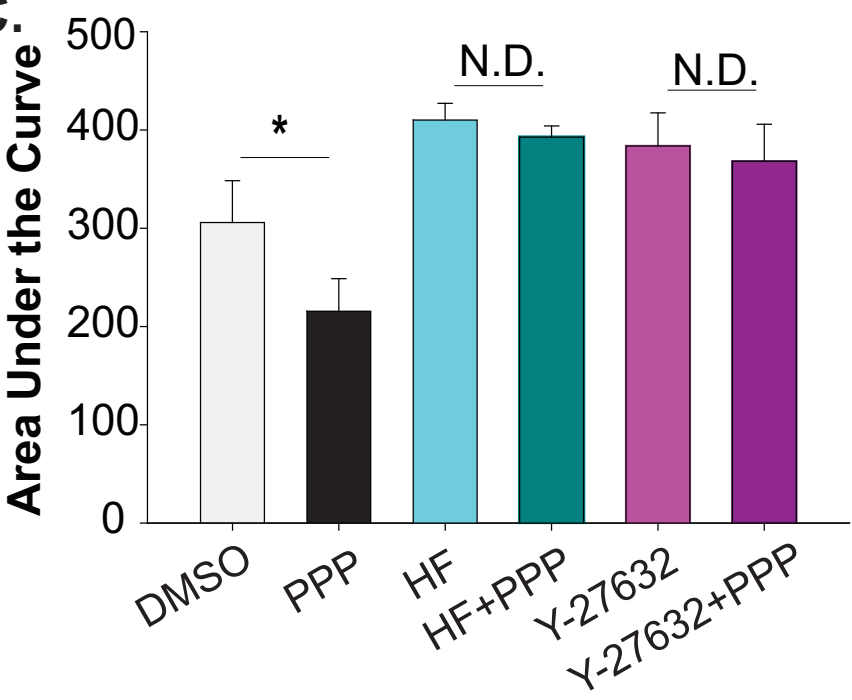




\section{FIGURE 5:}

A.

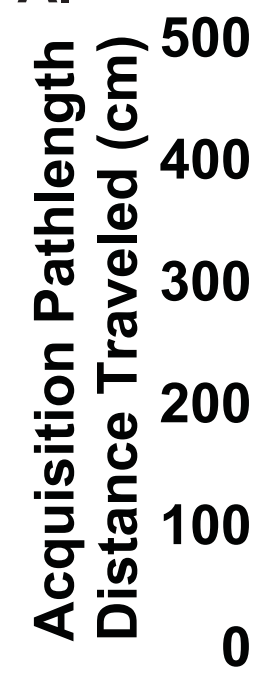

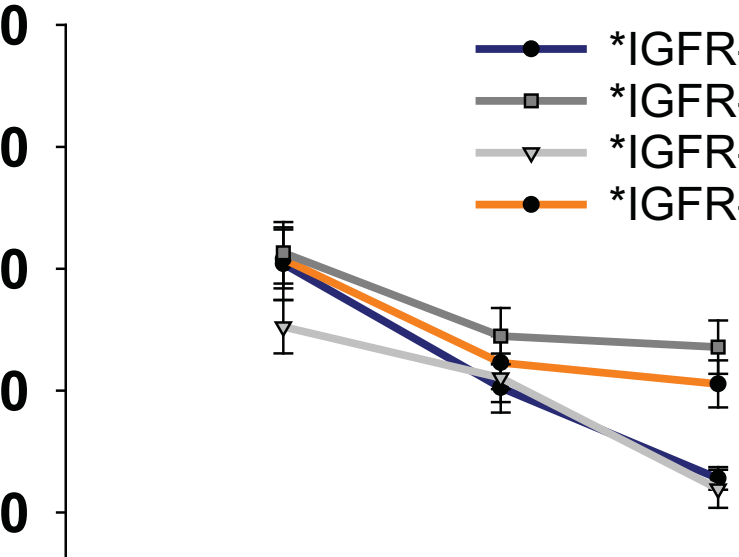

B.

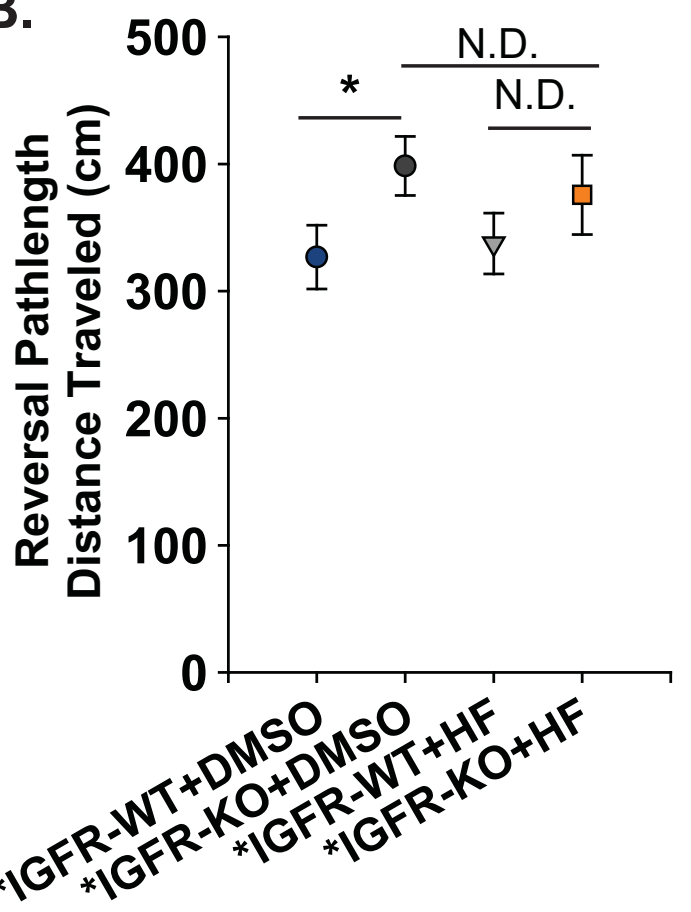

C.

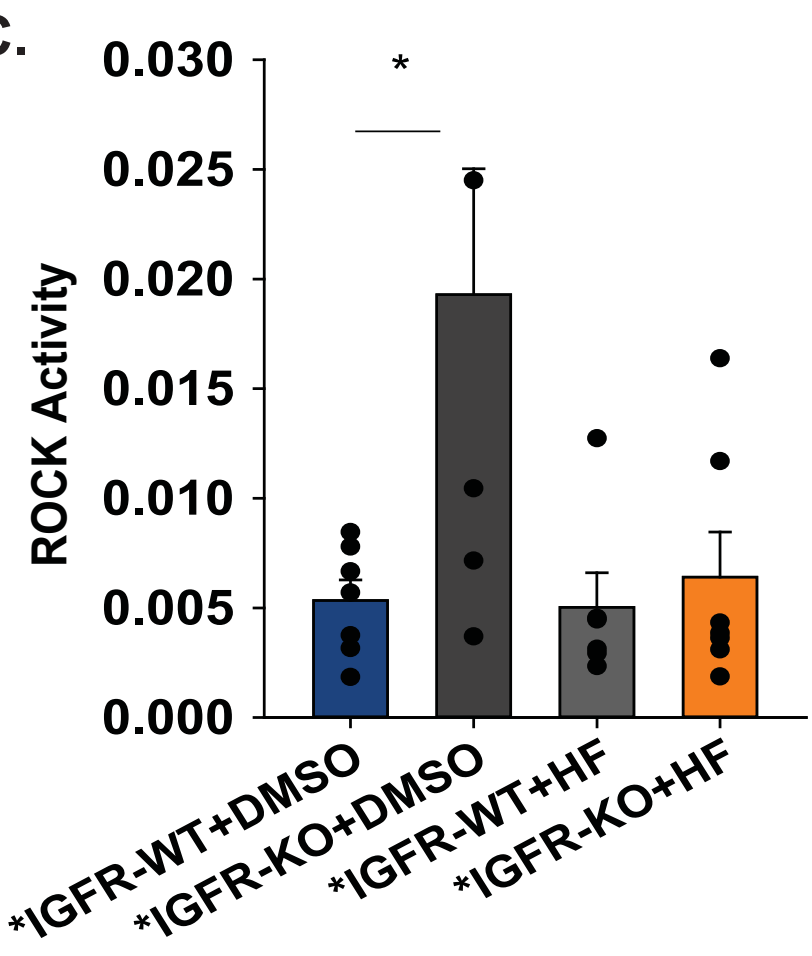

D.

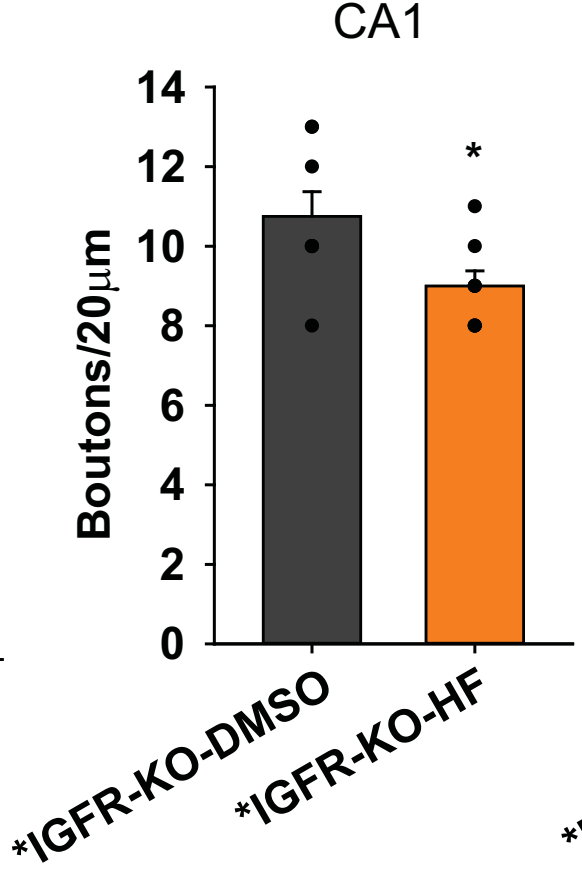

E. Dentate Gyrus

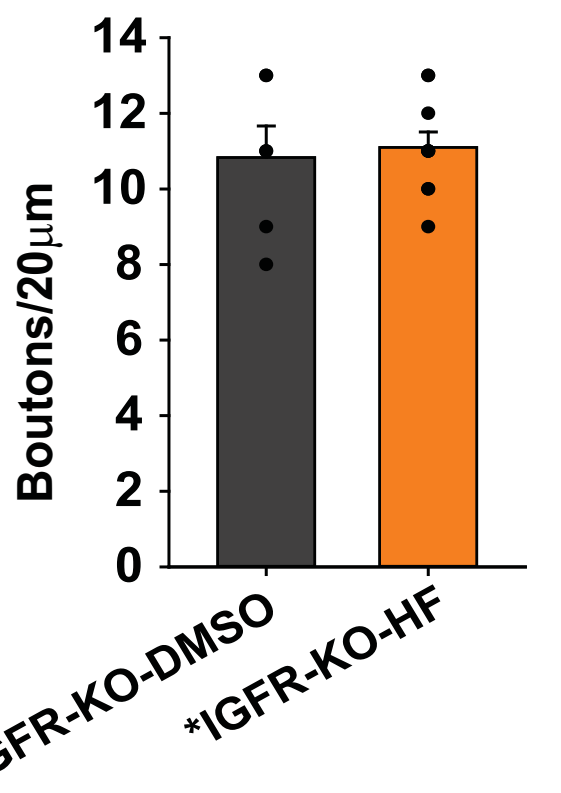




\section{Supplemental Information- Hayes et al}

Supplemental Figure 1: Sex-specific alterations in learning and memory and neuron structure with hippocampal neuron IGFR deficiency. (A) Average pathlength traveled and errors made during radial arm maze acquisition in non-surgical control, surgical control (*IGFR-WT), and surgical knockout (*IGFR-KO) male mice. (B) Average pathlength traveled and errors made by female mice during the acquisition and reversal phase of radial arm water maze $(n=9,10)$. (C) Working memory, novel location discrimination index, and novel object discrimination index in female *IGFR-WT and *IGFR-KO $(n=9,10)$. (D) The average of synaptic boutons across 20 microns of dedrites in the CA of female *IGFR-WT and *IGFR-KO (n=8,8). (E) Top: Representative images of syanptophysin and GAPDH western blots; bottom the average ratio of sypnatophysin to GAPDH protein expression in male *IGFR-WT and *IGFR-KO $(n=3,5)$. (F) Top: representative image of PSD95 and GAPDH western blots; bottom: the average ratio of PSD95 to GAPDH protein expression in female *IGFR-WT and *IGFR-KO hippocampal tissue $(n=3,5)$. \#Treatment denotes a difference between groups $(\mathrm{p}<0.05$; Two-way ANOVA repeated measures). All data represent mean +/- SEM. Abbreviations: F1,F2,F3 indicates Familiarization trial number. N.L novel location, N.O. novel object.

Supplemental Figure 2: (A) Average mRNA expression of RhoA, ROCK1, and ROCK2 in neurons deficient in IGF-1R (AAV-mediated Cre recombinase in igfr/f/f neurons). (B) Average ROCK2 protein expression in hippocampal neurons deficient in IGF-1R. (C) Top: Representative image of RhoA western blot, Bottom: Average RhoA and phosphorylated RhoA levels (Ser188) in cultured neurons treated with $0.5 \mu \mathrm{M}$ PPP or DMSO control.

Supplemental Figure 3: Spatial learning and memory in RAWM of males that received pharmacological treatments. (A) The average total number of errors between *IGFR-WT and *IGFR-KO that received DMSO and HF in the acquisition phase of RAWM (n=14). (B) The average total number of errors in the reversal phase of RAWM between four treatment groups $(n=14)$. (C) The average number of boutons on dendrites in the dentate gyrus of the hippocampus in *IGFR-KOs that received treatments, DMSO and HF $(n=7,10)$. All data are represented as mean $+/$ - SEM. Asterisk denotes $\mathrm{p}<0.05$ vs. respective control (t-test). 
Supplemental Table 1: Antibodies used with RRIDs. Abbreviations: BSA bovine serum albumin, TBST Tris Buffered Saline with Tween, ON overnight, $h$ hour

\begin{tabular}{|c|c|c|c|c|c|}
\hline Antibody & $\begin{array}{l}\text { Host } \\
\text { Type }\end{array}$ & $\begin{array}{c}\text { Source/ Catalog } \\
\text { Number. }\end{array}$ & Blocking Solution & $\begin{array}{c}\text { Dilution, } \\
\text { Incubation }\end{array}$ & RRID \\
\hline Synaptophysin & Rabbit & $\begin{array}{l}\text { ABCAM } \\
\text { ab32127 }\end{array}$ & $5 \%$ BSA+1XTBST & 1:1000; ON & AB_2286949 \\
\hline PSD95 & Mouse & $\begin{array}{l}\text { Thermo-Fisher } \\
\text { MA1-045 }\end{array}$ & $5 \%$ BSA+1XTBST & 1:2000; ON & AB_325399 \\
\hline GAPDH & Rat & $\begin{array}{l}\text { BioLegend Cat. } \\
\text { No. } 607901\end{array}$ & $5 \%$ BSA+1XTBST & $1: 5000 ; \mathrm{ON}$ & AB_2734502 \\
\hline $\begin{array}{l}\text { Anti-Cofilin } \\
\text { (phospho S3) }\end{array}$ & Rabbit & $\begin{array}{l}\text { ABCAM } \\
\text { ab12866 }\end{array}$ & $5 \%$ BSA+1XTBST & 1:1000; ON & AB_299488 \\
\hline Anti-Cofilin & Mouse & $\begin{array}{l}\text { ABCAM } \\
\text { ab54532 }\end{array}$ & $5 \%$ BSA+1XTBST & 1:1000; ON & AB_941092 \\
\hline Phospho-MAPK & Rabbit & $\begin{array}{l}\text { Cell Signaling } \\
\text { 4307S }\end{array}$ & 5\%BSA+1XTBST & 1:2000; ON & AB_2315112 \\
\hline MAPK & Mouse & $\begin{array}{l}\text { Cell Signaling } \\
\text { 9107S }\end{array}$ & $5 \%$ BSA+1XTBST & $1: 2000 ; \mathrm{ON}$ & AB_10695739 \\
\hline RhoA & Mouse & $\begin{array}{l}\text { ABCAM } \\
\text { Ab54835 }\end{array}$ & $5 \% B S A+1 X T B S T$ & $1: 500 ; 2 \mathrm{~h}$ & AB_945224 \\
\hline Actin & Mouse & $\begin{array}{l}\text { EnCor } \\
\text { MCA-5J11 }\end{array}$ & $5 \%$ BSA+1XTBST & 1:1000; ON & AB_2572218 \\
\hline Phospho-RhoA & Rabbit & $\begin{array}{l}\text { ABCAM } \\
\text { Ab41435 }\end{array}$ & 5\%BSA+1XTBST & 1:1000; ON & AB_777708 \\
\hline ROCK1 & Rabbit & $\begin{array}{l}\text { ABCAM } \\
\text { Ab45171 } \\
\end{array}$ & $5 \% B S A+1 X T B S T$ & 1:500; ON & AB_2182005 \\
\hline ROCK2 & Rabbit & $\begin{array}{l}\text { ABCAM } \\
\text { Ab71598 } \\
\end{array}$ & 5\%BSA+1XTBST & 1:1000; ON & AB_1566688 \\
\hline 800CW anti-rabbit & Goat & $\begin{array}{l}\text { Licor } \\
925-3211 \\
\end{array}$ & $5 \%$ BSA+1XTBST & 1:10000; $1 \mathrm{~h}$ & \\
\hline 680RD anti-rabbit & Goat & $\begin{array}{l}\text { Licor } \\
925-68071\end{array}$ & $5 \% B S A+1 X T B S T$ & $1: 10000 ; 1 \mathrm{~h}$ & \\
\hline 800CW anti-rabbit & Goat & $\begin{array}{l}\text { Licor } \\
925-82210\end{array}$ & $5 \% \mathrm{BSA}+1 \mathrm{XTBST}$ & $1: 10000 ; 1 \mathrm{~h}$ & \\
\hline 680RD anti-mouse & Goat & $\begin{array}{l}\text { Licor } \\
925-68070\end{array}$ & $5 \%$ BSA+1XTBST & 1:10000; 1h & \\
\hline
\end{tabular}


Supplemental Table 2: A summarized table of statistical comparisons denoting figure, details of comparisons, sample size, and $\mathrm{P}$ values. Abbreviations: $H F$ Hydroxyfasudil, $P P P$ picropodophyllin toxin, DMSO dimethylsulfoxide, $W T$ *IGFR-WT wildtype control that received AAV9-Syn-GFP, KO knockout of neuronal IGF-1R (*IGFR-KO) that received AV9-Syn-Cre, $N L$ Novel Location, NO Novel Object, DI discrimination index, GHR growth hormone receptor, InsR Insulin Receptor, IGF1 Insulin-Like Growth Factor-1, IGFR Insulin-like growth factor receptor, ROCK Rho Kinase

\begin{tabular}{|c|c|c|c|}
\hline Figure Number & Comparison Details & $\mathrm{n}$ & p-value \\
\hline Figure 1B & IGF1R hippocampal mRNA expression WT vs KO & 9 & 0.0334 \\
\hline Figure 1B & IGF1 hippocampal mRNA expression WT vs KO & 9 & 0.185 \\
\hline Figure 1B & GHR hippocampal mRNA expression WT vs KO & 9 & 0.201 \\
\hline Figure 1B & InsR hippocampal mRNA expression WT vs KO & 9 & 0.391 \\
\hline Figure 1C & Male Pathlength (Treatment) & 10,11 & 0.0002098 \\
\hline Figure 1C & Male Pathlength (Day) & 10,11 & 0.9847203 \\
\hline Figure 1D & Male Reversal Pathlength & 10,11 & 0.00172 \\
\hline Figure $1 \mathrm{E}$ & Male Error (Treatment) & 10,11 & 0.01864 \\
\hline Figure $1 \mathrm{E}$ & Male Error (Day) & 10,11 & 0.65185 \\
\hline Figure $1 \mathrm{~F}$ & Male Reversal Total Errors & 10,11 & 0.0111 \\
\hline Figure 1G & NO Pathlength-Males (Phase) & 9 & 0.01372 \\
\hline Figure 1G & NO Pathlength-Males (Treatment) & 9 & 0.16256 \\
\hline Figure $1 \mathrm{H}$ & NL DI Males & 8,9 & 0.762 \\
\hline Figure 1I & NO DI Males & 8,9 & 0.810 \\
\hline Figure 2B & CA1 Bouton of Hippocampus- Males & 9,12 & 0.000215 \\
\hline Figure 2E & Area under the curve PPP curve $-n=$ total neurons traced & $60-80$ & 0.006 \\
\hline Figure $3 \mathrm{~A}$ & Hippocampal Phosphorylated MAPK/MAPK & 8,13 & 0.009 \\
\hline Figure 3B & ROCK activity in hippocampal tissue & 6,7 & 0.025 \\
\hline Figure 3C & Hippocampal Phosphocofilin/Cofilin & 7,8 & 0.081 \\
\hline Figure 3D & ROCK activity culture (Genetic Treatment) & 11,12 & 0.004 \\
\hline Figure 3D & ROCK activity culture (IGF-1 vs Vehicle treatment) & 11,12 & 0.433 \\
\hline Figure 3D & ROCK activity culture (Genetic vs IGF-1/Vehicle treatment) & 11,12 & 0.286 \\
\hline Figure 3E & In vitro ROCK activity with PPP & 10 & 0.021 \\
\hline Figure 3E & In vitro ROCK activity with IGF-1 & 5,10 & 0.705 \\
\hline Figure $3 \mathrm{~F}$ & In vitro ROCK2 protein expression with PPP & 4 & 0.16 \\
\hline Figure $3 \mathrm{H}$ & In vitro GTP-Bound RhoA with PPP & 4 & 0.551 \\
\hline Figure 4C & Area under the curve- PPP vs DMSO & 60,80 & 0.002 \\
\hline Figure 4C & Area under the curve- HF vs HF PPP & 47,60 & - \\
\hline Figure 4C & Area under the curve- Y-27632 vs Y-27632 PPP & 77,80 & - \\
\hline Figure 4C & Area under the curve- HF vs Y-27632 & 60,77 & - \\
\hline Figure 5A & DMSO WT-KO Pathlength (Day) & 14 & $1.583 \mathrm{e}-10$ \\
\hline Figure 5A & DMSO WT-KO Pathlength (Injection) & 14 & 0.44855 \\
\hline Figure 5A & HF WT-KO Pathlength (Day) & 14 & $2.335 \mathrm{e}-06$ \\
\hline Figure 5A & HF WT-KO Pathlength (Injection) & 14 & 0.8372 \\
\hline Figure 5A & HF-DMSO WT Pathlength (Day) & 14 & $2.561 \mathrm{e}-13$ \\
\hline Figure 5A & HF-DMSO WT Pathlength (Injection) & 14 & 0.1693 \\
\hline Figure 5A & HF-DMSO KO Pathlength (Day) & 14 & $9.316 \mathrm{e}-05$ \\
\hline Figure 5A & HF-DMSO KO Pathlength (Injection) & 14 & 0.7968 \\
\hline Figure 5B & Reversal Pathlength (Genetic Treatment) & 14 & 0.034 \\
\hline Figure 5B & Reversal Pathlength- (Injection Treatment) & 14 & 0.518 \\
\hline Figure 5C & ROCK activity- WT vs KO DMSO & 6,7 & 0.025 \\
\hline Figure 5C & ROCK activity- WT vs KO HF & 6,7 & - \\
\hline Figure 5C & ROCK activity- KO DMSO vs KO HF & 6,7 & 0.036 \\
\hline
\end{tabular}




\begin{tabular}{|c|c|c|c|}
\hline Figure 5D & Hippocampal Boutons: CA1 & 8 & 0.0302 \\
\hline Figure 5E & Hippocampal Boutons: DG & 7,10 & 0.751 \\
\hline Supplemental Figure 1A & Male Pathlength WT vs No Surgery & 6,10 & 0.05 \\
\hline Supplemental Figure 1A & Male Pathlength KO vs No Surgery & 6,11 & - \\
\hline Supplemental Figure 1A & Male Pathlength WT vs KO & 10,11 & 0.006 \\
\hline Supplemental Figure 1B & Female Pathlength (Treatment) & 9,10 & 0.0738 \\
\hline Supplemental Figure 1B & Female Pathlength (Day) & 9,10 & $9.391 \mathrm{e}-13$ \\
\hline Supplemental Figure 1B & Female Reversal Pathlength & 9,10 & 0.491 \\
\hline Supplemental Figure 1B & Female Error (Treatment) & 9,10 & 0.09046 \\
\hline Supplemental Figure 1B & Female Error (Day) & 9,10 & $1.65 \mathrm{e}-08$ \\
\hline Supplemental Figure 1B & Female Reversal Total Errors & 9,10 & 0.784 \\
\hline Supplemental Figure 1C & NO Pathlength- Females (Phase) & 10 & $7.00 \mathrm{e}-15$ \\
\hline Supplemental Figure 1C & NO Pathlength- Females (Treatment) & 10 & $7.610 \mathrm{e}-07$ \\
\hline Supplemental Figure 1C & NL DI Females & 9 & 0.962 \\
\hline Supplemental Figure 1C & NO DI Females & 9 & 0.267 \\
\hline Supplemental Figure 1D & CA1 Bouton of Hippocampus- Females & 16 & 0.181 \\
\hline Supplemental Figure 1E & Synaptophysin/GAPDH- Males & 3,5 & 0.662 \\
\hline Supplemental Figure 1E & PSD95/GAPDH- Males & 3,5 & 0.278 \\
\hline Supplemental Figure 2A & In vitro RhoA mRNA expression with KO & 4 & 0.205 \\
\hline Supplemental Figure 2A & In vitro RhoA mRNA expression with KO & 4 & 0.152 \\
\hline Supplemental Figure 2A & In vitro RhoA mRNA expression with KO & 4 & 0.069 \\
\hline Supplemental Figure 2B & In vitro ROCK2 protein expression with KO & 6 & 0.117 \\
\hline Supplemental Figure 2C & In vitro RhoA protein expression with PPP & 3 & 0.526 \\
\hline Supplemental Figure 2C & In vitro Phospho-RhoA Expression with PPP & 7 & 1.00 \\
\hline Supplemental Figure 3A & DMSO WT-KO Error (Day) & 14 & 7.84e-07 \\
\hline Supplemental Figure 3A & DMSO WT-KO Error (Injection) & 14 & 0.5386 \\
\hline Supplemental Figure 3A & HF WT-KO Error (Day) & 14 & 0.01094 \\
\hline Supplemental Figure 3A & HF WT-KO Error (Injection) & 14 & 0.35391 \\
\hline Supplemental Figure 3A & HF-DMSO WT Error (Day) & 14 & $5.138 \mathrm{e}-07$ \\
\hline Supplemental Figure 3A & HF-DMSO WT Error (Injection) & 14 & 0.0770 \\
\hline Supplemental Figure 3A & HF-DMSO KO Error (Day) & 14 & 0.0118 \\
\hline Supplemental Figure 3A & HF-DMSO KO Error (Injection) & 14 & 0.9659 \\
\hline Supplemental Figure 3B & Reversal Total Errors DMSO Treatment & 14 & 0.0201 \\
\hline Supplemental Figure 3B & Reversal Total Errors HF Treatment & 14 & 0.041 \\
\hline
\end{tabular}


A.
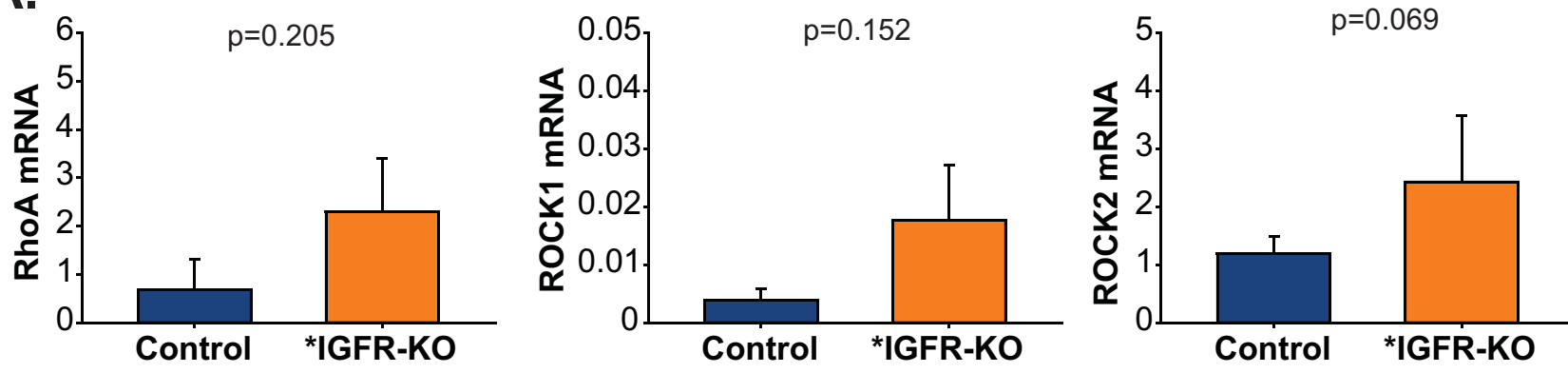

B.

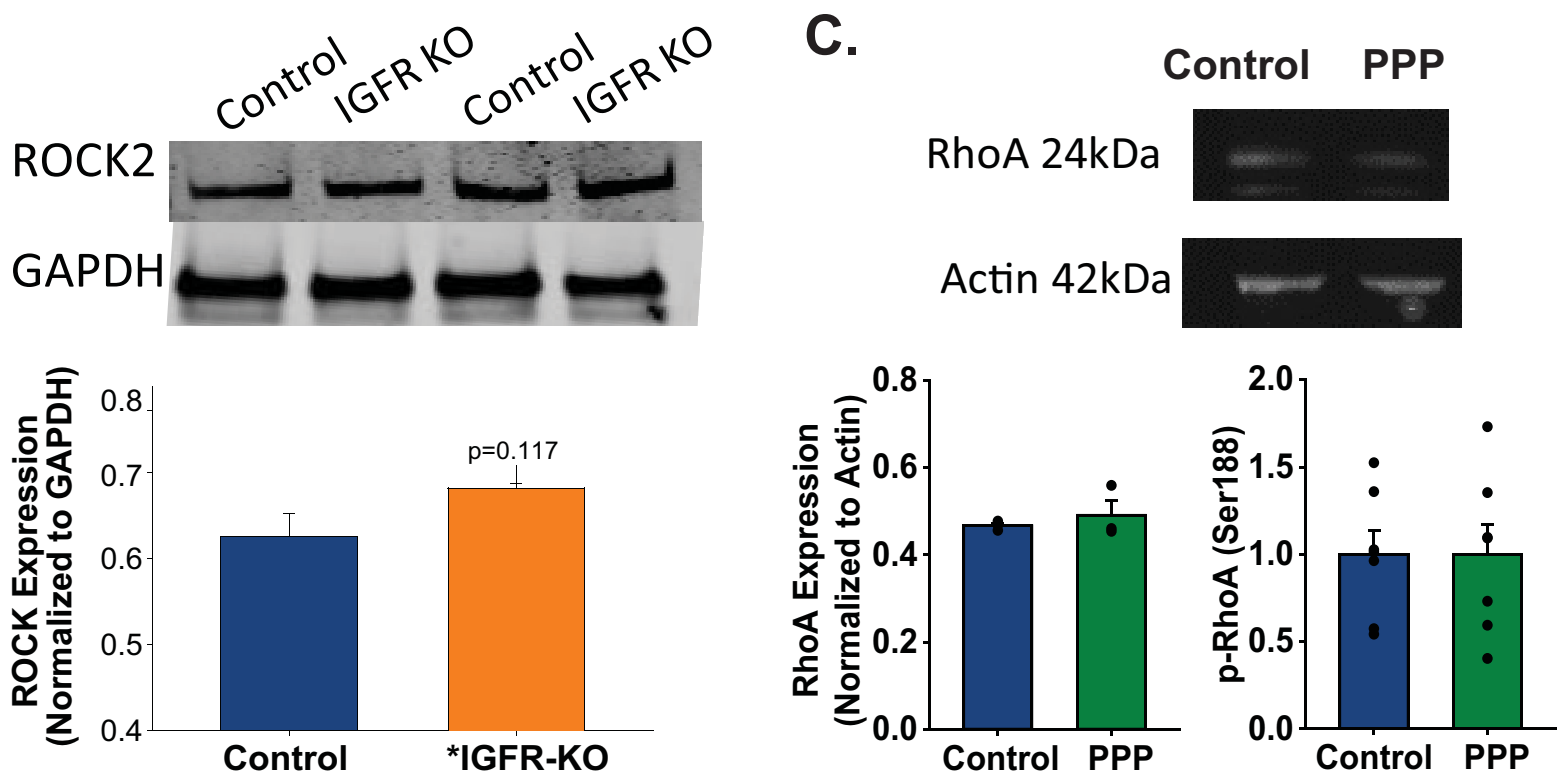


A.

B.
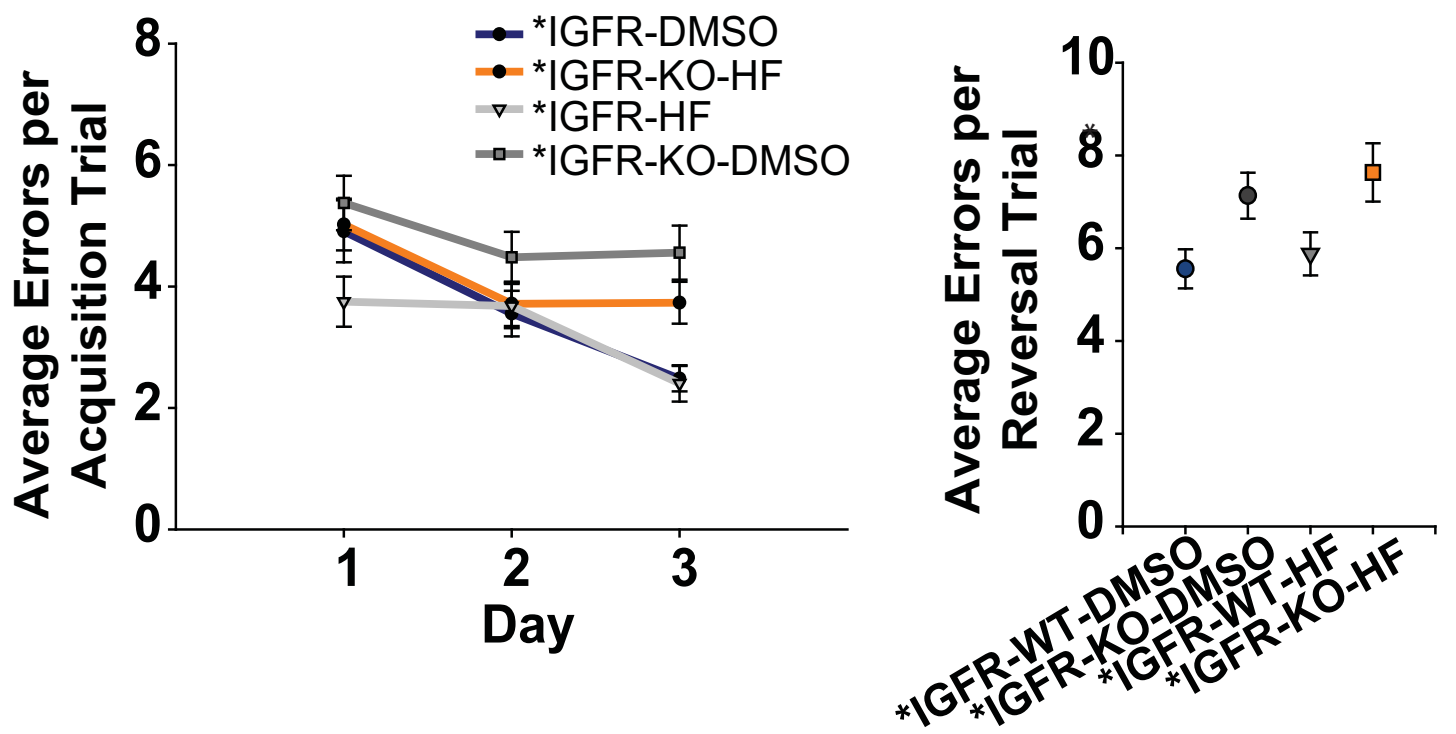